\title{
Prognostic role of STAT3 in solid tumors: a systematic review and meta-analysis
}

\author{
Pin Wu ${ }^{1,4, *}$, Dang Wur ${ }^{2,4, *}$, Lufeng Zhao ${ }^{1}$, Lijian Huang ${ }^{1}$, Gang Shen ${ }^{1}$, Jian Huang ${ }^{3,4}$, \\ Ying Chai ${ }^{1}$ \\ ${ }^{1}$ Department of Thoracic Surgery, Second Affiliated Hospital, Zhejiang University School of Medicine, Zhejiang University, \\ Hangzhou, 310009, China \\ ${ }^{2}$ Department of Radiation Oncology, Second Affiliated Hospital, Zhejiang University School of Medicine, Zhejiang University, \\ Hangzhou, 310009, China \\ ${ }^{3}$ Department of Surgical Oncology, Second Affiliated Hospital, Zhejiang University School of Medicine, Zhejiang University, \\ Hangzhou, 310009, China \\ ${ }^{4}$ Cancer Institute, Second Affiliated Hospital, Zhejiang University School of Medicine, Zhejiang University, Hangzhou, 310009, China \\ *These authors contributed equally to this work
}

Correspondence to: Ying Chai, e-mail: chaiy@126.com

Pin Wu, e-mail: doctorwp@163.com

Keywords: STAT3, solid tumors, prognosis, overall survival, disease free survival

Received: October 12, 2015

Accepted: February 13, 2016

Published: March 03, 2016

\section{ABSTRACT}

Accumulated studies have provided controversial evidences of the association between signal transducer and activator of transcription proteins 3 (STAT3) expression and survival of human solid tumors. To address this inconsistency, we performed a meta-analysis with 63 studies identified from PubMed, Medline and EBSCO. We found STAT3 overexpression was significantly associated with worse 3-year overall survival (OS) $(O R=2.06,95 \% \mathrm{CI}=1.57$ to $2.71, P<0.00001)$ and 5 -year OS (OR $=2.00,95 \%$ $\mathrm{CI}=1.53$ to $2.63, P<0.00001$ ) of human solid tumors. Similar results were observed when disease free survival (DFS) were analyzed. Subgroup analysis showed that elevated STAT3 expression was associated with poor prognosis of gastric cancer, lung cancer, gliomas, hepatic cancer, osteosarcoma, prostate cancer, pancreatic cancer but better prognosis of breast cancer. The correlation between STAT3 and survival of solid tumors was related to its phosphorylated state. High expression level of STAT3 was also associated with advanced tumor stage. In conclusion, elevated STAT3 expression is associated with poor survival in most solid tumors. STAT3 is a valuable biomarker for prognosis prediction and a promising therapeutic target in human solid tumors.

\section{INTRODUCTION}

Signal transducer and activator of transcription proteins 3 (STAT3) is well demonstrated to play a crucial role in tumor development and cancer-related inflammation [1]. STAT3 is also linked to inflammationrelated oncogenesis initiated by genetic alterations and environmental factors [2-4], and is constitutively activated in various cancers $[5,6]$. Persistent activation of STAT3 is involved in promoting tumor cell proliferation, survival, tumor invasion, angiogenesis and immunosuppression, inducing and maintaining a pro-carcinogenic inflammatory microenvironment [7]. Growing studies identified novel tumor-promoting functions of STAT3 in mitochondria metabolism [8], drug resistance $[9,10]$, epigenetic regulation [11], cancer stem cells $[12,13]$ and premetastatic niches $[14,15]$. Given the pivotal role in tumor development, STAT3 represents an attractive therapeutic target for solid tumors. Recently, accumulating studies have demonstrated STAT3-targeted therapy could effectively restrain tumor development in various solid tumors [16-21]. However, the prognostic value of STAT3 overexpression in human solid tumors is still controversial.

A plenty of studies showed that elevated STAT3 expression in tumor tissue was correlated with poor survival of patients with various solid tumors such as gastric 
cancer [22-29], lung cancer [30-37], gliomas [38-42], colorectal cancer [43], ovarian cancer [44], cervical cancer [45], hepatocellular carcinoma [46, 47], melanoma [48], esophageal cancer [49], osteosarcoma [50, 51], pancreatic cancer $[52,53]$, thymic epithelial tumor [54], astrocytomas [55], lingual squamous cell carcinoma [56], nasopharyngeal carcinoma [57], prostate cancer [58], renal cell carcinoma [59] and Wilms' tumor [60]. However, other studies reported that overexpression of STAT3 was correlated with favorable outcome of patients with breast cancer [61-65], gastric cancer [66], lung cancer [67-69], colorectal cancer [70, 71] and melanoma [72].

Therefore, we carried out a meta-analysis combining available evidences to evaluate the prognostic value of STAT3 expression in solid tumors. We also evaluated whether the clinical outcome of patients with solid tumors differed between STAT3 phosphorylation state and between different tumor types. This meta-analysis intended to assess the role of STAT3 in relation to survival in solid tumors, thereby supporting more rational development of therapeutic strategies against STAT3.

\section{RESULTS}

\section{Search results and study characteristics}

Sixty-three studies with a total of 9449 patients were included (Figure 1). Characteristics of included studies are shown in Table 1. Eleven studies evaluated lung cancer [30-37, 67-69], nine evaluated gastric cancer [22-29, 66], five evaluated breast cancer [61-65], five evaluated gliomas [38-42], four evaluated colorectal cancer $[43,70,71,73]$, three evaluated ovarian cancer $[44,74,75]$, three evaluated cervical cancer $[45,76,77]$, two evaluated hepatocellular carcinoma [46, 47], two evaluated melanoma $[48,72]$, two evaluated esophageal cancer $[49,78]$, two evaluated osteosarcoma $[50,51]$, two evaluated pancreatic cancer [52,53], two evaluated thymic epithelial tumours [54,79], two oral cancer [80, 81], and one each evaluated astrocytomas [55], chordoma [82], head and neck squamous cell carcinoma [83], lingual squamous cell carcinoma [56], nasopharyngeal carcinoma [84], pharyngeal cancer [57], prostate cancer [58], renal cell carcinoma [59], and Wilms' tumor [60]. Of these 63 studies, 20 studies evaluated STAT3, 37 studies evaluated p-STAT3, and 6 studies evaluated both STAT3 and p-STAT3. As for the region, 39 studies were conducted in Asia, 13 studies in America, 10 studies in Europe, and 1 study in Austria.

\section{Evaluation and expression of STAT3}

Antibodies, detection and definition method, and cut-off values of STAT3 expression used in the included studies is summarized in Table 2. Diverse antibodies were used for the assessment of STAT3 expression by
IHC. For anti-STAT3 antibody, three studies used clone sc-8019, one study each used clone RB-9237, F-2, sc-7179, 79D7, 124H6, and sixteen studies did not report the antibody clone. For anti-p-STAT3 antibody, eight studies used clone D3A7, four studies used clone sc-7993, two studies used clone 9131 , one study each used sc-483, sc-8001, sc-8059, ZP-0647, and twenty studies did not report the antibody clone. The median expression of STAT3 in solid tumors was $47.79 \%$, range from $19.65 \%$ to $94.66 \%$.

\section{Association of STAT3 with OS}

The combined analysis of 54 studies showed that STAT3 overexpression in tumor tissue was associated with worse 3 -year $\mathrm{OS}$ of solid tumors ( $\mathrm{OR}=2.06,95 \%$ $\mathrm{CI}=1.57$ to $2.71, P<0.00001)$ (Figure 2). There was significant heterogeneity among studies (Cochran's Q $P<0.00001, \mathrm{I}^{2}=81 \%$ ), so we conducted meta-regression analysis and subgroup meta-analysis to investigate the possible source of the heterogeneity among studies.

In the stratified analysis by tumor types, STAT3 expression was associated with worse 3 -year OS of gastric cancer $(\mathrm{OR}=4.06,95 \% \mathrm{CI}=1.86$ to $8.89, P=0.0004)$, lung cancer $(\mathrm{OR}=2.22,95 \% \mathrm{CI}=1.31$ to $3.77, P=0.003)$, gliomas $(\mathrm{OR}=4.10,95 \% \mathrm{CI}=1.50$ to $11.23, P=0.006)$, hepatic cancer $(\mathrm{OR}=3.75,95 \% \mathrm{CI}=1.71$ to 8.21 , $P=0.001)$, osteosarcoma $(\mathrm{OR}=3.94,95 \% \mathrm{CI}=1.83$ to $8.51, P=0.0005)$ and prostate cancer $(\mathrm{OR}=11.08,95 \%$ $\mathrm{CI}=1.24$ to $98.96, P=0.03$ ) (Figure 3 ). There was no significant association between STAT3 expression and 3-year OS of colorectal cancer, ovarian cancer, pancreatic cancer, cervical cancer, melanoma and thymic epithelial tumor (Supplementary Figure S1). Interestingly, STAT3 overexpression was associated with favorable 3-year OS of breast cancer (OR $=0.51,95 \% \mathrm{CI}=0.35$ to 0.74 , $P=0.0004$ ) (Supplementary Figure S2).

Meta-regression analysis showed that publication year, country, gender and NOS score did not contribute to the heterogeneity (data not shown).

Analysis of 49 studies showed STAT3 expression was also associated with worse 5-year OS (OR $=2.00,95 \%$ $\mathrm{CI}=1.53$ to $2.63, P<0.00001$ ) (Figure 4 ) of solid tumors. There was also high heterogeneity among studies for 5-year OS (Cochran's Q $P<0.00001, \mathrm{I}^{2}=82 \%$ ), so we conducted subgroup meta-analysis.

Subgroup analysis showed that STAT3 expression was associated with worse 5 -year OS of gastric cancer $(\mathrm{OR}=5.48,95 \% \mathrm{CI}=2.14$ to $14.01, P=0.0004)$, hepatic cancer $(\mathrm{OR}=2.48,95 \% \mathrm{CI}=1.41$ to 4.35 , $P=0.002)$, osteosarcoma ( $\mathrm{OR}=4.84,95 \% \mathrm{CI}=2.23$ to $10.50, P<0.0001)$, pancreatic cancer $(\mathrm{OR}=9.71,95 \%$ $\mathrm{CI}=1.80$ to $52.41, P=0.008)$ and prostate cancer $(\mathrm{OR}=8.35,95 \% \mathrm{CI}=1.81$ to38.51, $P=0.007)($ Figure 5$)$. There was no significant association between STAT3 expression and the 5-year OS of colorectal cancer, lung cancer, ovarian cancer, cervical cancer, melanoma and 
thymic epithelial tumor (Supplementary Figure S3). STAT3 overexpression was associated with favorable 5 -year OS of breast cancer $(\mathrm{OR}=0.57,95 \% \mathrm{CI}=0.37$ to $0.89, P=0.01$ ) (Supplementary Figure S4).

Twenty studies evaluated STAT3, 38 studies evaluated p-STAT3 and 5 studies evaluated both STAT3 and p-STAT3. Our result showed that both STAT3 and p-STAT3 overexpression were associated with worse OS of solid tumors. However, elevated p-STAT3 $(\mathrm{OR}=2.45,95 \% \mathrm{CI}=1.73$ to $3.46, P<0.00001)$ expression in tumor tissue seemed to be more significantly associated with worse 3-year OS than STAT3 expression $(\mathrm{OR}=1.72,95 \% \mathrm{CI}=1.10$ to 2.70 , $P=0.02$ ) (Supplementary Figure S5). Similar result was observed for 5-year OS analysis (Supplementary Figure S6). A subgroup meta-analysis of studies evaluated both STAT3 and p-STAT3 shown that p-STAT3 expression was associated with worse 3-year and 5-year
OS of solid tumor, but not STAT3 (Supplementary Figure S7).

We also evaluated the correlation between STAT3 overexpression and the TNM stage of tumor. High expression level of STAT3 was significantly associated with advanced TNM stage $(\mathrm{OR}=0.42,95 \% \mathrm{CI}=0.31$ to $0.58, P<0.00001$ ) (Figure 6).

Next, we conducted subgroup analysis according to STAT3 expression level. Results showed STAT3 expression was associated with poor 3-year OS in the studies using cutoff values of $10 \%-30 \%(\mathrm{OR}=3.61,95 \%$ $\mathrm{CI}=2.42$ to $5.39, P<0.00001)$ and $50 \%(\mathrm{OR}=2.14,95 \%$ $\mathrm{CI}=1.29$ to $3.57, P=0.003$ ) (Figure 7) to determine STAT3 positivity. Similar result was observed in 5-year OS (Supplementary Figure S6). However, the studies used cutoff value of STAT3 overexpression as more than 1\%$6 \%$ tumor cells positive was not associated with 3 -year and 5-year OS of solid tumors.

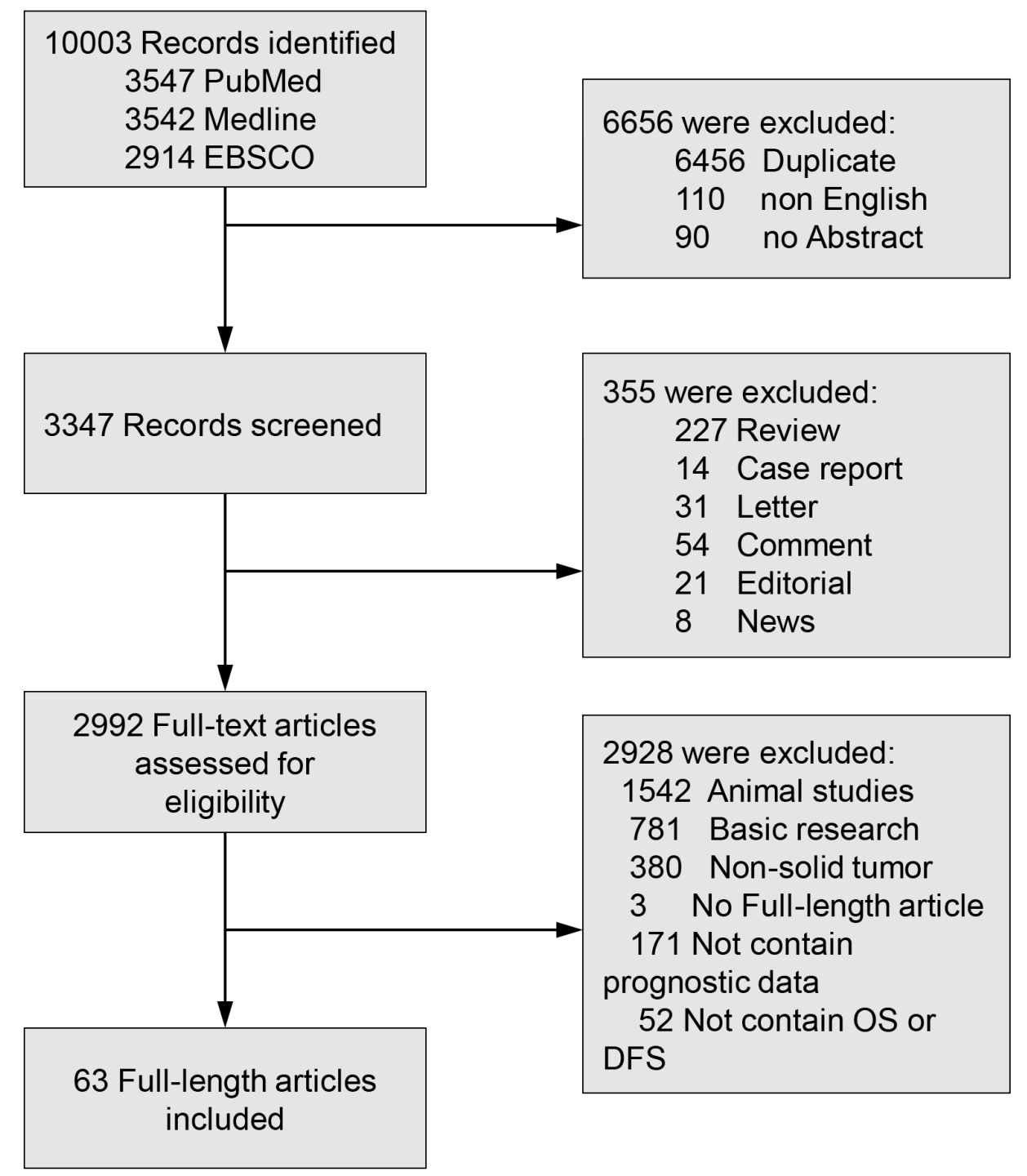

Figure 1: Flow diagram of study selection. STAT3: Signal transducer and activator of transcription protein 3; OS: overall survival; DFS: disease-free survival. 
Table 1: Characteristics of studies included in the meta-analysis

\begin{tabular}{|c|c|c|c|c|c|c|c|c|c|c|c|}
\hline References & Country & Type of cancer & $\begin{array}{l}\text { Patient } \\
\text { No. }\end{array}$ & $\begin{array}{l}\text { Age, } \\
\text { median } \\
\text { (range) }\end{array}$ & $\begin{array}{c}\text { Male/ } \\
\text { Female }\end{array}$ & Stage & $\begin{array}{c}\text { Follow-Up, } \\
\text { months } \\
\text { (Range) }\end{array}$ & $\begin{array}{l}\text { STAT3 (+/-) } \\
\text { NO. }\end{array}$ & $\begin{array}{c}\text { 3-year } \\
\text { OS } \\
(+/-) \%\end{array}$ & $\begin{array}{c}\text { 5-year } \\
\text { OS } \\
(+/-) \%\end{array}$ & $\begin{array}{l}\text { NOS } \\
\text { Score }\end{array}$ \\
\hline \multicolumn{12}{|c|}{ Studies including OS } \\
\hline $\begin{array}{l}\text { Abou- } \\
\text { Ghazal, M., } \\
\text { et al. (2008) }\end{array}$ & USA & Gliomas & 128 & $\begin{array}{c}44 \\
(4-91)\end{array}$ & NR & II-IV & NR & $65 / 63$ & $36.7 / 48.9$ & $34.7 / 41.7$ & 7 \\
\hline $\begin{array}{l}\text { Ai, T., et al. } \\
(2012)\end{array}$ & China & NSCLC & 65 & NR & $50 / 15$ & I-IV & $29.9 \pm 15.7$ & $47 / 18$ & $49.8 / 77.9$ & NR & 8 \\
\hline $\begin{array}{l}\text { Birner, P., } \\
\text { etal. (2010) }\end{array}$ & Bulgaria & Gliomas & 111 & $58.0 \pm 11.6$ & $57 / 54$ & NR & $11.1 \pm 0.8$ & $65 / 46$ & $0 / 17.9$ & NR & 7 \\
\hline $\begin{array}{l}\text { Chang, K. C., } \\
\text { et al. (2006) }\end{array}$ & China & TET & 118 & $\begin{array}{c}52.7 \\
(25-77)\end{array}$ & $65 / 53$ & I-IV & NR & $38 / 80$ & $58.7 / 56.7$ & $32.9 / 30$ & 7 \\
\hline $\begin{array}{l}\text { Chatterjee, } \\
\text { Devasis., } \\
\text { et al. (2008) }\end{array}$ & USA & $\mathrm{GC}$ & 143 & $\begin{array}{c}71.1 \\
(31-96)\end{array}$ & $75 / 68$ & IA-IV & $\begin{array}{c}34 \\
(12-180)\end{array}$ & $40 / 103$ & $45.1 / 70.1$ & $0 / 60.7$ & 8 \\
\hline $\begin{array}{l}\text { Chen, C. C., } \\
\text { et al. (2010) }\end{array}$ & China & $\mathrm{NC}$ & 95 & NR & NR & I-IV & $\begin{array}{c}112.8 \\
(31.2-240)\end{array}$ & $34 / 61$ & $39 / 61.2$ & $31.3 / 53$ & 6 \\
\hline $\begin{array}{l}\text { Chen, H. H., } \\
\text { et al. (2012) }\end{array}$ & China & $\mathrm{CC}$ & 165 & $\begin{array}{c}69 \\
(29-89)\end{array}$ & $0 / 165$ & I-IV & NR & $36 / 129$ & $53.8 / 60$ & $47.3 / 48.5$ & 8 \\
\hline $\begin{array}{l}\text { Cortas, T., } \\
\text { et al. (2007) }\end{array}$ & USA & NSCLC & 145 & $70(40-88)$ & $64 / 81$ & I-III & $\begin{array}{c}35 \\
(4-85)\end{array}$ & $50 / 84$ & $70 / 60.7$ & $55.5 / 52$ & 7 \\
\hline $\begin{array}{l}\text { Deng, J. Y., } \\
\text { et al. (2010) }\end{array}$ & China & $\mathrm{GC}$ & 53 & $\begin{array}{c}55 \\
(31-78)\end{array}$ & $37 / 16$ & I-IV & $\begin{array}{c}38 \\
(2-108)\end{array}$ & $26 / 27$ & $11.5 / 85.2$ & $3.8 / 85.2$ & 8 \\
\hline $\begin{array}{l}\text { Deng, J., } \\
\text { et al. (2013) }\end{array}$ & China & $\mathrm{GC}$ & 114 & NR & $76 / 38$ & NR & NR & $89 / 25$ & $24.6 / 80$ & $9.9 / 50.6$ & 7 \\
\hline $\begin{array}{l}\text { Denley, } \\
\text { S. M., } \\
\text { et al. (2013)- } \\
\text { Tyr705 }\end{array}$ & UK & PDA & 86 & NR & $43 / 43$ & NR & 22 & $29 / 57$ & $9 / 21.9$ & $0 / 11$ & 7 \\
\hline $\begin{array}{l}\text { Denley, } \\
\text { S. M., } \\
\text { et al. (2013)- } \\
\text { Ser727 }\end{array}$ & UK & PDA & 86 & NR & $43 / 43$ & NR & 22 & $30 / 56$ & $83 / 22.2$ & $0 / 11.2$ & 7 \\
\hline $\begin{array}{l}\text { Dolled-F. M., } \\
\text { et al. (2003)- } \\
\text { M1-C }\end{array}$ & USA & $\mathrm{BC}$ & 286 & NR & $0 / 286$ & NR & NR & $198 / 88$ & $94.6 / 87.3$ & $85.5 / 80$ & 6 \\
\hline $\begin{array}{l}\text { Dolled-F. M., } \\
\text { et al. (2003)- } \\
\text { M1-N }\end{array}$ & USA & $\mathrm{BC}$ & 286 & NR & $0 / 286$ & NR & NR & $66 / 220$ & $94.6 / 92.1$ & $93.1 / 81.2$ & 6 \\
\hline $\begin{array}{l}\text { Dolled-F. M., } \\
\text { et al. (2003)- } \\
\text { M2-C }\end{array}$ & USA & $\mathrm{BC}$ & 286 & NR & $0 / 286$ & NR & NR & $56 / 229$ & $92.5 / 92.5$ & $86.7 / 83.7$ & 6 \\
\hline $\begin{array}{l}\text { Dolled-F. M., } \\
\text { et al. (2003)- } \\
\text { M2-N }\end{array}$ & USA & $\mathrm{BC}$ & 286 & NR & $0 / 286$ & NR & NR & $124 / 161$ & $95.7 / 89.9$ & $91.3 / 78.7$ & 6 \\
\hline $\begin{array}{l}\text { Galleges } \\
\text { Ruiz, M. I., } \\
\text { et al. (2009) }\end{array}$ & USA & NSCLC & 178 & NR & $127 / 51$ & I-III & NR & $51 / 111$ & $54.5 / 45$ & $48.8 / 36.5$ & 7 \\
\hline $\begin{array}{l}\text { Gordziel, } \\
\text { C., et al. } \\
\text { (2013)-C }\end{array}$ & Germany & $\mathrm{CRC}$ & 414 & NR & NR & I-III & $37(0-146)$ & $132 / 282$ & $82.8 / 70.6$ & $74 / 61$ & 6 \\
\hline $\begin{array}{l}\text { Gordziel, } \\
\text { C., et al. } \\
\text { (2013)-N }\end{array}$ & Germany & $\mathrm{CRC}$ & 414 & NR & NR & I-III & $37(0-146)$ & $124 / 290$ & $78.6 / 72$ & $71.2 / 62.1$ & 6 \\
\hline
\end{tabular}




\begin{tabular}{|c|c|c|c|c|c|c|c|c|c|c|c|}
\hline $\begin{array}{l}\text { Haura, Eric } \\
\text { B., et al. } \\
(2005)\end{array}$ & USA & NSCLC & 176 & $69(45-84)$ & $97 / 79$ & I & $72(36-108)$ & $94 / 82$ & $77.4 / 74.3$ & $57.8 / 52.2$ & 8 \\
\hline $\begin{array}{l}\text { Hbibi, A. } \\
\text { Tadlaoui., } \\
\text { et al. (2008)- } \\
\text { M1 }\end{array}$ & France & $\mathrm{CRC}$ & 126 & 68.1 & NR & I-IV & NR & $62 / 38$ & NR & $61.3 / 49.2$ & 7 \\
\hline $\begin{array}{l}\text { Hbibi, A. } \\
\text { Tadlaoui., } \\
\text { et al. (2008)- } \\
\text { M2 }\end{array}$ & France & $\mathrm{CRC}$ & 126 & 68.1 & NR & I-IV & NR & $27 / 73$ & NR & $59.1 / 55.6$ & 7 \\
\hline $\begin{array}{l}\text { Horiguchi, } \\
\text { Akio., et al. } \\
(2002)\end{array}$ & Japan & $\mathrm{RCC}$ & 48 & $63(24-85)$ & $39 / 9$ & I-IV & $15.9(1-101$ & $24 / 24$ & $53.5 / 89.7$ & $53.5 / 89.7$ & 7 \\
\hline $\begin{array}{l}\text { Huang, C., } \\
\text { et al. (2012) }\end{array}$ & China & PDA & 71 & $67(40-80)$ & $50 / 21$ & I-IV & $33.7(3-60)$ & $39 / 32$ & $0 / 24.3$ & $0 / 14.2$ & 8 \\
\hline $\begin{array}{l}\text { Jia, Yanfei., } \\
\text { et al. (2013) }\end{array}$ & China & $\mathrm{GC}$ & 48 & $66(45-83)$ & $34 / 14$ & I-IV & NR & $19 / 29$ & $54.4 / 86.7$ & $12.7 / 47.6$ & 7 \\
\hline $\begin{array}{l}\text { Kim, D. Y., } \\
\text { et al. (2009) }\end{array}$ & Korea & GC & 71 & NR & $48 / 23$ & I-IV & $30(11-83)$ & $27 / 44$ & $59 / 86.4$ & $40 / 81.8$ & 6 \\
\hline $\begin{array}{l}\text { Kim, Yeon- } \\
\text { Joo., et al. } \\
(2011)\end{array}$ & Korea & $\mathrm{NC}$ & 38 & $48(25-74)$ & $30 / 8$ & I-IV & $\begin{array}{c}43.7 \\
(0.71-60)\end{array}$ & $10 / 28$ & NR & $41 / 77$ & 8 \\
\hline $\begin{array}{l}\text { Kusaba, T., } \\
\text { et al. (2006) }\end{array}$ & Japan & $\mathrm{CRC}$ & 108 & $\begin{array}{c}65.6 \\
(44-86)\end{array}$ & $66 / 42$ & I-IV & NR & $62 / 46$ & 61.6/90.1 & $48.8 / 90.1$ & 8 \\
\hline $\begin{array}{l}\text { Lee, I., et al. } \\
(2012)\end{array}$ & USA & Melanoma & 299 & $\begin{array}{c}56 \\
(13-85)\end{array}$ & $212 / 87$ & IV & NR & $236 / 63$ & $44.1 / 41.8$ & $22.3 / 25.4$ & 7 \\
\hline $\begin{array}{l}\text { Lee, J., et al. } \\
(2009)\end{array}$ & China & $\mathrm{GC}$ & 303 & NR & $206 / 97$ & II-III & $\begin{array}{c}61.5 \\
(12-134)\end{array}$ & $79 / 224$ & $68.4 / 78.4$ & $59.5 / 70.5$ & 7 \\
\hline $\begin{array}{l}\text { Li, Chao., } \\
\text { et al. (2013) }\end{array}$ & China & TET & 80 & $\begin{array}{c}46.5 \\
(19-70)\end{array}$ & $47 / 33$ & I-IV & NR & $36 / 44$ & $70.1 / 100$ & $46.8 / 97.6$ & 7 \\
\hline $\begin{array}{l}\text { Lin, G. S., } \\
\text { et al. (2014) }\end{array}$ & China & Gliomas & 90 & 55 (18-79). & $54 / 36$ & NR & $\begin{array}{c}46.4 \\
(1.2-109.6)\end{array}$ & $73 / 17$ & $14 / 31.4$ & NR & 8 \\
\hline $\begin{array}{l}\text { Mano, Y., } \\
\text { et al. (2013) }\end{array}$ & Japan & $\mathrm{HC}$ & 101 & NR & $81 / 20$ & NR & NR & $36 / 65$ & $71.3 / 84.9$ & $60.7 / 84.7$ & 8 \\
\hline $\begin{array}{l}\text { Min, Hao., } \\
\text { et al. (2009)- } \\
\text { M1 }\end{array}$ & China & $\mathrm{OC}$ & 50 & $50.6(22-73)$ & $0 / 50$ & I-IV & NR & $44 / 6$ & $53.5 / 75.1$ & $29 / 0$ & 6 \\
\hline $\begin{array}{l}\text { Min, Hao., } \\
\text { et al. (2009)- } \\
\text { M2 }\end{array}$ & China & $\mathrm{OC}$ & 50 & $50.6(22-73)$ & $0 / 50$ & I-IV & NR & $29 / 21$ & $35 / 88.1$ & $0 / 58.7$ & 6 \\
\hline $\begin{array}{l}\text { Monnien, F., } \\
\text { et al. (2010) }\end{array}$ & France & $\mathrm{CRC}$ & 104 & $66(37-80)$ & $76 / 28$ & NR & $13(8-48)$ & $39 / 65$ & $82.2 / 78$ & $71.8 / 65.5$ & 7 \\
\hline $\begin{array}{l}\text { Pectasides, } \\
\text { Eirini., et al. } \\
(2010)-1\end{array}$ & Greece & HNSC & 107 & NR & $87 / 20$ & I-IV & $64(1-120)$ & $23 / 47$ & $90.4 / 45.9$ & $72.4 / 38.3$ & 7 \\
\hline $\begin{array}{l}\text { Pectasides, } \\
\text { Eirini., et al. } \\
(2010)-2\end{array}$ & Greece & HNSC & 107 & NR & $87 / 20$ & I-IV & $64(1-120)$ & $12 / 25$ & NR & $68.8 / 51.4$ & 7 \\
\hline $\begin{array}{l}\text { Piperi, } \\
\text { Christina., } \\
\text { et al. (2011) }\end{array}$ & Greece & Gliomas & 97 & $59(19-82)$ & $60 / 37$ & II-IV & $63(3-180)$ & $89 / 8$ & $0 / 14$ & NR & 6 \\
\hline $\begin{array}{l}\text { Rosen, D. G., } \\
\text { et al. (2006) }\end{array}$ & USA & $\mathrm{OC}$ & 303 & $58.2(20-86)$ & $0 / 303$ & I-IV & 52 & $215 / 88$ & $49.9 / 60$ & $32.1 / 49.1$ & 8 \\
\hline $\begin{array}{l}\text { Ryu, } \\
\text { Keinosuke., } \\
\text { et al. (2010) }\end{array}$ & USA & Osteosarcoma & 51 & $20.5(5-61)$ & $38 / 13$ & NR & NR & $31 / 20$ & $48.5 / 75$ & $35.2 / 75$ & 8 \\
\hline
\end{tabular}




\begin{tabular}{|c|c|c|c|c|c|c|c|c|c|c|c|}
\hline $\begin{array}{l}\text { Schoppmann, } \\
\text { S. F., et al. } \\
(2012)\end{array}$ & Austria & $\mathrm{EC}$ & 324 & 63 & $252 / 72$ & NR & NR & $144 / 180$ & $32.6 / 57.6$ & $24.9 / 53$ & 7 \\
\hline $\begin{array}{l}\text { Sheen-Chen, } \\
\text { et al. (2008) }\end{array}$ & China & $\mathrm{BC}$ & 102 & $48.2(26-76)$ & $0 / 102$ & I-III & NR & $27 / 75$ & NR & $59 / 77.2$ & 6 \\
\hline $\begin{array}{l}\text { Slinger, E., } \\
\text { et al. (2010) }\end{array}$ & Sweden & Gliomas & 21 & NR & NR & NR & NR & $7 / 14$ & $0 / 13.6$ & NR & 8 \\
\hline $\begin{array}{l}\text { Sonnenblick, } \\
\text { A., et al. } \\
(2012)\end{array}$ & Israel & $\mathrm{BC}$ & 125 & NR & $0 / 125$ & NR & 50 & $35 / 90$ & $100 / 91$ & $94.4 / 76.6$ & 6 \\
\hline $\begin{array}{l}\text { Sonnenblick, } \\
\text { A., et al. } \\
\text { (2013)-1 }\end{array}$ & Israel & $\mathrm{BC}$ & 375 & 50 & $0 / 375$ & NR & NR & $47 / 82$ & $97.9 / 96.3$ & $94 / 94$ & 7 \\
\hline $\begin{array}{l}\text { Sonnenblick, } \\
\text { A., et al. } \\
(2013)-2\end{array}$ & Israel & $\mathrm{BC}$ & 375 & 50 & $0 / 375$ & NR & NR & $184 / 150$ & 99/91.9 & $94.1 / 80.2$ & 7 \\
\hline $\begin{array}{l}\text { Takemoto, S., } \\
\text { et al. (2009) }\end{array}$ & Japan & $\mathrm{CC}$ & 125 & 47 (19-77) & $0 / 125$ & I-II & NR & $71 / 54$ & $80.9 / 96.8$ & $78.5 / 94.5$ & 7 \\
\hline $\begin{array}{l}\text { Tam, L., et al. } \\
(2007)-\mathrm{N}\end{array}$ & UK & $\mathrm{PC}$ & 50 & $70(64-73)$ & $50 / 0$ & NR & $29.5(15-54)$ & $22 / 28$ & $72.8 / 92.5$ & $57.9 / 84.2$ & 8 \\
\hline $\begin{array}{l}\text { Tam, L., et al. } \\
\text { (2007)-C }\end{array}$ & UK & $\mathrm{PC}$ & 50 & $70(64-73)$ & $50 / 0$ & NR & $29.5(15-54)$ & $19 / 31$ & $58.1 / 100$ & $42.5 / 92.7$ & 8 \\
\hline $\begin{array}{l}\text { van Cruijsen, } \\
\text { H., et al. } \\
(2009)\end{array}$ & USA & NSCLC & 164 & 64.5 & NR & I-III & NR & $116 / 48$ & $49 / 58.7$ & $33.1 / 50.3$ & 7 \\
\hline $\begin{array}{l}\text { Wang, M., } \\
\text { et al. (2011) }\end{array}$ & China & NSCLC & 208 & $59.8(35-76)$ & NR & I-III & $67(1-78.2)$ & $128 / 80$ & $53.9 / 73.2$ & $24.7 / 39.8$ & \\
\hline $\begin{array}{l}\text { Wang, Y. C., } \\
\text { et al. (2011) }\end{array}$ & China & Osteosarcoma & 76 & NR & $25 / 51$ & NR & 37 & $36 / 40$ & $25.8 / 60.4$ & $25.8 / 60.4$ & 6 \\
\hline $\begin{array}{l}\text { Wang, Y., } \\
\text { et al. (2011) }\end{array}$ & China & Gliomas & 68 & $45(15-68)$ & $41 / 27$ & NR & $51(1-72)$ & $47 / 21$ & $0 / 15.4$ & NR & 7 \\
\hline $\begin{array}{l}\text { Woo, S., } \\
\text { et al. (2011) }\end{array}$ & Korea & GC & 285 & 54.4 & $193 / 92$ & I-IV & $39.7(4-84)$ & $101 / 179$ & 79/61.6 & $74.9 / 54.5$ & 7 \\
\hline $\begin{array}{l}\text { Wu, Z.S., } \\
\text { et al. (2011) }\end{array}$ & China & Melanoma & 90 & NR & $52 / 38$ & I-IV & NR & $51 / 39$ & $80.5 / 97.6$ & $50.8 / 76.7$ & 8 \\
\hline $\begin{array}{l}\text { Xiong, Hua., } \\
\text { et al. (2012)- } \\
\text { M1 }\end{array}$ & China & GC & 262 & $59.3(23-79)$ & $176 / 86$ & I-IV & $90(2-273)$ & $248 / 14$ & $44 / 64.3$ & $28.4 / 42.9$ & 8 \\
\hline $\begin{array}{l}\text { Xiong, Hua., } \\
\text { et al. (2012)- } \\
\text { M2 }\end{array}$ & China & $\mathrm{GC}$ & 262 & $59.3(23-79)$ & $176 / 86$ & I-IV & $90(2-273)$ & $136 / 126$ & $25.3 / 65.5$ & $11.5 / 47.3$ & 8 \\
\hline $\begin{array}{l}\text { Yakata, } \\
\text { Yuichi., et al. } \\
\text { (2007) }\end{array}$ & Japan & GC & 111 & $68.9(38-89)$ & $63 / 48$ & NR & 120 & $55 / 56$ & $37.7 / 83.4$ & $37.7 / 78.6$ & 8 \\
\hline $\begin{array}{l}\text { Yamashita, } \\
\text { H., et al. } \\
(2006)\end{array}$ & Japan & $\mathrm{BC}$ & 506 & NR (22-91) & $0 / 506$ & NR & NR & $206 / 300$ & $92 / 87.9$ & $86 / 81.6$ & 7 \\
\hline $\begin{array}{l}\text { Yang, C., } \\
\text { et al. (2013) }\end{array}$ & USA & $\mathrm{OC}$ & 49 & $61(41-87)$ & $0 / 49$ & I-IV & NR & $25 / 24$ & $70.6 / 73.4$ & $33.5 / 57.6$ & 7 \\
\hline $\begin{array}{l}\text { Yang, Cao., } \\
\text { et al. (2009) }\end{array}$ & USA & Chordoma & 70 & $59.5(29-88)$ & $51 / 19$ & NR & $\begin{array}{c}16.8 \\
(0.8-69.2)\end{array}$ & $35 / 35$ & $82.5 / 90.2$ & $73.1 / 90.2$ & 8 \\
\hline $\begin{array}{l}\text { Yin, Z., et al. } \\
(2012)\end{array}$ & China & NSCLC & 76 & NR & $48 / 28$ & I-IV & NR & $42 / 34$ & $49.6 / 59.3$ & $41.8 / 57.9$ & 7 \\
\hline $\begin{array}{l}\text { Yu, Y., et al. } \\
(2015)\end{array}$ & China & NSCLC & 82 & NR & $48 / 34$ & I-IV & NR & $76 / 24$ & $28.7 / 76.2$ & $20.3 / 48.3$ & 8 \\
\hline
\end{tabular}




\begin{tabular}{|c|c|c|c|c|c|c|c|c|c|c|c|}
\hline $\begin{array}{l}\text { Zhang, C. H., } \\
\text { et al. (2012)- } \\
\text { M1 }\end{array}$ & China & $\mathrm{HC}$ & 100 & $55.1(28-77)$ & $80 / 20$ & I-IV & 15.4 & $72 / 28$ & $53.5 / 57.8$ & $14 / 32.2$ & 8 \\
\hline $\begin{array}{l}\text { Zhang, C. H., } \\
\text { et al. (2012)- } \\
\text { M2 }\end{array}$ & China & $\mathrm{HC}$ & 100 & $55.1(28-77)$ & $80 / 20$ & I-IV & 15.4 & $58 / 42$ & $35.5 / 81.2$ & $19 / 25.7$ & 8 \\
\hline $\begin{array}{l}\text { Zhang, L. J., } \\
\text { et al. (2013) }\end{array}$ & China & Wilms' tumor & 58 & $31(3-132)$ & $38 / 20$ & I-IV & $\geq 78$ & $17 / 41$ & $45.6 / 72.1$ & $45.6 / 72.1$ & 7 \\
\hline $\begin{array}{l}\text { Zhao, X., } \\
\text { et al. (2012)- } \\
\text { M1 }\end{array}$ & China & SCLC & 128 & NR & $66 / 62$ & I-IV & $67(1-78.2)$ & $71 / 57$ & $29.7 / 81.6$ & $0 / 9.9$ & 7 \\
\hline $\begin{array}{l}\text { Zhao, X., } \\
\text { et al. (2012)- } \\
\text { M2 }\end{array}$ & China & SCLC & 128 & NR & $66 / 62$ & I-IV & $67(1-78.2)$ & $62 / 66$ & $43.4 / 62.3$ & $0 / 3.9$ & 7 \\
\hline $\begin{array}{l}\text { Zhao, Yan., } \\
\text { et al. (2012) }\end{array}$ & China & LSCC & 163 & NR & NR & I-IV & NR & $100 / 63$ & $75 / 86.7$ & $41.8 / 78.6$ & 8 \\
\hline $\begin{array}{l}\text { Studies } \\
\text { including } \\
\text { DFS }\end{array}$ & & & & & & & & & & & \\
\hline $\begin{array}{l}\text { Choi, Chel } \\
\text { Hun., et al. } \\
(2010)\end{array}$ & Korea & $\mathrm{CC}$ & 29 & NR & $0 / 29$ & I-II & NR & $20 / 9$ & $49.9 / 84.6$ & $49.9 / 84.6$ & 8 \\
\hline $\begin{array}{l}\text { Lee, J., et al. } \\
(2009)\end{array}$ & China & $\mathrm{GC}$ & 303 & NR & $206 / 97$ & III-III & $\begin{array}{c}61.5 \\
(12-134)\end{array}$ & $79 / 224$ & $61.7 / 73.7$ & $58.3 / 67.7$ & 7 \\
\hline $\begin{array}{l}\text { Li, X., et al. } \\
(2015)\end{array}$ & China & NSCLC & 164 & NR & $115 / 40$ & I-III & NR & $107 / 57$ & $57.4 / 87.6$ & NR & 8 \\
\hline $\begin{array}{l}\text { Macha, } \\
\text { Muzafar A., } \\
\text { et al. (2011) }\end{array}$ & Canada & Oral cancer & 94 & NR & $70 / 24$ & I-IV & NR & $63 / 31$ & $18.6 / 53.9$ & $7.1 / 53.9$ & 7 \\
\hline $\begin{array}{l}\text { Mano, Y., } \\
\text { et al. (2013) }\end{array}$ & Japan & $\mathrm{HC}$ & 101 & NR & $81 / 20$ & NR & NR & $36 / 65$ & $12.5 / 55.6$ & $12.5 / 31.5$ & 8 \\
\hline $\begin{array}{l}\text { Schoppmann, } \\
\text { S. F., et al. } \\
(2012)\end{array}$ & Austria & $\mathrm{EC}$ & 324 & 63 & $252 / 72$ & NR & NR & $144 / 180$ & $25.8 / 48.3$ & $20.2 / 47.2$ & 7 \\
\hline $\begin{array}{l}\text { Takemoto, S., } \\
\text { et al. (2009) }\end{array}$ & Japan & $\mathrm{CC}$ & 125 & 47 (19-77) & $0 / 125$ & I-II & NR & $71 / 54$ & $82 / 97.8$ & $78 / 95.3$ & 7 \\
\hline $\begin{array}{l}\text { Wang, Y. C., } \\
\text { et al. (2011) }\end{array}$ & China & Osteosarcoma & 76 & NR & $25 / 51$ & NR & 37 & $36 / 40$ & $33.8 / 67.1$ & $24.9 / 56.3$ & 6 \\
\hline $\begin{array}{l}\text { Yamashita, } \\
\text { H., et al. } \\
(2006)\end{array}$ & Japan & $\mathrm{BC}$ & 506 & NR (22-91) & $0 / 506$ & NR & NR & $206 / 300$ & $83.2 / 74.7$ & $72.7 / 65.3$ & 7 \\
\hline $\begin{array}{l}\text { Zhang, L. J., } \\
\text { et al. (2013) }\end{array}$ & China & Wilms' tumor & 58 & $31(3-132)$ & $38 / 20$ & I-IV & $\geq 78$ & $17 / 41$ & $31.4 / 74.1$ & $31.4 / 74.1$ & 7 \\
\hline
\end{tabular}

M1: Marker1, STAT3; M2: Marker 2, p-STAT3; N: nuclear expression; C: cytoplasmic expression; 1: Cohort 1; 2: Cohort 2; NSCLC: Non-Small Cell Lung Cancer; GC: Gastric cancer; CRC: Colorectal Cancer; BC: Breast cancer; CC: Cervical Carcinoma; OC: Ovarian Carcinoma; PDA: Pancreatic Ductal Adenocarcinoma; TET: Thymic Epithelial Tumours; RCC: Renal Cell Carcinoma; HC: Hepatocellular Carcinoma; HNSCC: Head and Neck Squamous Cell Carcinoma; EC: Esophageal Cancer; OSCC: Oral Squamous Cell Carcinoma; SCLC: Small Cell Lung Cancer; NC: Nasopharyngeal carcinoma; LSCC: Lingual Squamous Cell Carcinoma; PC: Prostate Cancer; NR: Not Reported; DFS: disease-free survival, STAT3: Signal transducer and activator of transcription protein 3; NOS: newcastle-Ottawa Scale; OS: overall survival. 
Table 2: Evaluation of human STAT3/p-STAT3 by IHC in the selected studies

\begin{tabular}{|c|c|c|c|c|}
\hline References & $\begin{array}{l}\text { Type of } \\
\text { cancer }\end{array}$ & Marker & Cutoff & Antibody (Clone) \\
\hline Abou-Ghazal, M., et al. (2008) & Gliomas & p-STAT3 & NR & anti-p-STAT3 (Tyr705), Cell Signaling Technology \\
\hline Ai, T., et al. (2012) & NSCLC & STAT3 & $\mathrm{IHC}>51 \%$ & anti-STAT3, Cell Signaling Technology \\
\hline Birner, P., etal. (2010) & Gliomas & p-STAT3 & $\mathrm{IHC} \geq 5 \%$ & anti-p-STAT3 (Tyr705), clone D3A7, Cell Signaling \\
\hline Chang, K. C., et al. (2006) & TET & STAT3 & $\mathrm{IHC} \geq 10 \%$ & $\begin{array}{l}\text { anti-Stat3 F-2: sc-8019, Santa Cruz Biotechnology, } \\
\text { Inc. }\end{array}$ \\
\hline Chatterjee, Devasis., et al. (2008) & $\mathrm{GC}$ & STAT3-nuclear & $\begin{array}{c}\text { IHC } \\
\text { scores } \geq 4\end{array}$ & anti-STAT3, Santa Cruz Biotechnology, Inc. \\
\hline Chen, H. H., et al. (2012) & $\mathrm{CC}$ & STAT3 & $\mathrm{IHC} \geq 20 \%$ & anti-STAT3, Santa Cruz Biotechnology, Inc. \\
\hline Chen, C. C., et al. (2010) & $\mathrm{NC}$ & p-STAT3 & $\mathrm{IHC}>10 \%$ & NR \\
\hline Choi, Chel Hun., et al. (2010) & $\mathrm{CC}$ & p-STAT3 & $\mathrm{IHC}>51 \%$ & anti-p-STAT3 (ser727), Santa Cruz Biotechnology \\
\hline Cortas, T., et al. (2007) & NSCLC & p-STAT3 & $\mathrm{IHC} \geq 5 \%$ & anti-p-STAT3 (sc-8059), Santa Cruz Biotechnology \\
\hline Deng, J. Y., et al. (2010) & GC & p-STAT3 & $\geq 10 \%$ & anti-p-STAT3 (sc-483) \\
\hline Deng, J., et al. (2013) & $\mathrm{GC}$ & p-STAT3 & $\mathrm{IHC}>25 \%$ & anti-p-STAT3, Santa, sc-8001-R \\
\hline \multirow[t]{2}{*}{ Denley, S. M., et al. (2013) } & PDA & p-STAT3 & $\mathrm{IHC} \geq 2 \%$ & anti-pStat3 Tyr 705, 9131,Cell Signaling Technology \\
\hline & & & & $\begin{array}{l}\text { anti-pStat3 (Ser 727), 9134, Cell Signaling } \\
\text { Technology }\end{array}$ \\
\hline Dobi, E., et al. (2013) & $\mathrm{CRC}$ & p-STAT3 & $\mathrm{IHC}>15 \%$ & anti-p-STAT3, sc-7993, Santa Cruz Biotechnology \\
\hline \multirow[t]{4}{*}{ Dolled-Filhart, M., et al. (2003) } & $\mathrm{BC}$ & STAT3-cytoplasmic & IHC score $\geq 1$ & anti-STAT3, Cell Signaling Technology \\
\hline & & \multicolumn{2}{|l|}{ STAT3-nuclear } & anti-STAT3, Cell Signaling Technology \\
\hline & & \multicolumn{2}{|l|}{ p-STAT3-cytoplasmic } & anti-p-STAT3 (Tyr 705), Cell Signaling Technology \\
\hline & & \multicolumn{2}{|l|}{ p-STAT3-nuclear } & anti-p-STAT3 (Tyr 705), Cell Signaling Technology \\
\hline Galleges Ruiz, M. I., et al. (2009) & NSCLC & p-STAT3-nuclear & $\begin{array}{c}\text { IHC } \\
\text { score }>210\end{array}$ & anti-p-STAT3 \\
\hline \multirow[t]{2}{*}{ Gordziel, C., et al. (2013) } & $\mathrm{CRC}$ & STAT3-cytoplasmic & IHC score $\geq 2$ & anti-STAT3: Stat3 (79D7), Cell Signaling Technology \\
\hline & & \multicolumn{2}{|l|}{ STAT3-nuclear } & \\
\hline Haura, Eric B., et al. (2005) & NSCLC & p-STAT3-nuclear & IHC score $\geq 1$ & anti-p-Stat3 (Tyr 705), Cell Signaling Technology \\
\hline \multirow[t]{2}{*}{ Hbibi, A. Tadlaoui., et al. (2008) } & CRC & p-STAT3 & IHC score $\geq 6$ & anti-P-STAT3 (Tyr 705), Cell Signaling Technology \\
\hline & & STAT3 & & anti-STAT3, Cell Signaling \\
\hline Horiguchi, Akio., et al. (2002) & $\mathrm{RCC}$ & p-STAT3 & $\mathrm{IHC} \geq 10 \%$ & anti-p-STAT3, (Tyr 705), Cell Signaling Technology \\
\hline Huang, C., et al. (2012) & PDA & p-STAT3 & $\mathrm{IHC} \geq 25 \%$ & anti-p-STAT3, Cell Signaling Technology \\
\hline Jia, Yanfei., et al. (2013) & $\mathrm{GC}$ & STAT3 & NR & anti-STAT3, Santa Cruz Biotechnology \\
\hline Kim, D. Y., et al. (2009) & $\mathrm{GC}$ & STAT3 & NR & anti-STAT3, Chemicon International \\
\hline Kim, Yeon-Joo., et al. (2011) & $\mathrm{NC}$ & STAT3 & $\mathrm{IHC} \geq 10 \%$ & anti-STAT3, Epitomics \\
\hline Kusaba, T., et al. (2006) & CRC & p-STAT3 & $\mathrm{IHC}>15 \%$ & anti-p-STAT3 (Tyr705), Santa Cruz Biotechnology \\
\hline Lee, I., et al. (2012) & Melanoma & p-STAT3 & $\mathrm{IHC} \geq 1 \%$ & anti-p-STAT3 (Tyr705), Santa Cruz Biotechnology \\
\hline Lee, J., et al. (2009) & $\mathrm{GC}$ & p-STAT3 & $\mathrm{IHC} \geq 1 \%$ & anti-p-STAT3 (Tyr705), Cell Signaling Technology \\
\hline Li, Chao., et al. (2013) & TET & STAT3 & $\mathrm{IHC}>10 \%$ & anti-STAT3, Santa Cruz Biotechnology \\
\hline Li, X., et al. (2015) & NSCLC & STAT3 & IHC score $\geq 4$ & anti-STAT3, Santa Cruz Biotechnology \\
\hline Lin, G. S., et al. (2014) & Gliomas & p-STAT3 & $\mathrm{IHC}>5 \%$ & anti-p-STAT3 (Tyr705), D3A7, Cell Signaling \\
\hline Macha, Muzafar A., et al. (2011) & Oral cancer & p-STAT3 & NR & anti-p-STAT3 (Tyr 705), Cell Signaling \\
\hline Mano, Y., et al. (2013) & $\mathrm{HC}$ & p-STAT3 & NR & anti-p-STAT3 (Tyr 705), D3A7, Cell Signaling \\
\hline Min, Hao., et al. (2009) & $\mathrm{OC}$ & STAT3 & $\mathrm{IHC} \geq 10 \%$ & anti-Stat3, (SC-8019), Santa Cruz Biotechnology \\
\hline
\end{tabular}




\begin{tabular}{|c|c|c|c|c|}
\hline & & p-STAT3 & $\mathrm{IHC} \geq 10 \%$ & $\begin{array}{l}\text { anti-p-Stat3 (Tyr 705), ZP-0647, Abzoom } \\
\text { Biotechnology }\end{array}$ \\
\hline Monnien, F., et al. (2010) & $\mathrm{CRC}$ & p-STAT3 & $\mathrm{IHC}>15 \%$ & anti-p-Stat3 (Tyr 705), sc-7993, Santa Cruz \\
\hline Pectasides, Eirini., et al. (2010) & HNSCC & STAT3-nuclear & NR & anti-Stat3, clone 124H6; Cell Signaling Technology \\
\hline Piperi, Christina., et al. (2011) & Gliomas & p-STAT3 & $\mathrm{IHC} \geq 6 \%$ & anti-p-STAT3 (Tyr 705), D3A7 XP, Cell Signaling \\
\hline Rosen, D. G., et al. (2006) & $\mathrm{OC}$ & p-STAT3 & $\mathrm{IHC}>10 \%$ & anti-p-Stat3, (SC-7993-R), Santa Cruz Biotechnology \\
\hline Ryu, Keinosuke., et al. (2010) & Osteosarcoma & p-STAT3 & $\mathrm{IHC}>51 \%$ & anti-p-STAT, Cell Signaling Technology \\
\hline $\begin{array}{l}\text { Schoppmann, Sebastian F., et al. } \\
\text { (2012) }\end{array}$ & $\mathrm{EC}$ & p-STAT3 & $\mathrm{IHC}>10 \%$ & anti-p-STAT3 (Tyr 705), D3A7, Cell Signaling \\
\hline Shah, N. G., et al. (2006) & OSCC & STAT3-nuclear & $\mathrm{IHC}>10 \%$ & anti-STAT3, Santa Cruz Biotechnology \\
\hline Slinger, E., et al. (2010) & Gliomas & p-STAT3 & $\mathrm{IHC}>30 \%$ & anti-p-STAT3, (Tyr 705), Cell Signaling \\
\hline $\begin{array}{l}\text { Sheen-Chen, Shyr-Ming., et al. } \\
(2008)\end{array}$ & $\mathrm{BC}$ & STAT3 & IHC score $\geq 3$ & anti-STAT3 (RB-9237), NeoMarkers \\
\hline Sonnenblick, A., et al. (2012) & $\mathrm{BC}$ & p-STAT3 & $\mathrm{IHC} \geq 25 \%$ & anti-p-STAT3, (Tyr 705), Cell Signaling \\
\hline Sonnenblick, A., et al. (2013) & $\mathrm{BC}$ & p-STAT3 & $\mathrm{IHC} \geq 10 \%$ & anti-p-STAT3, (Tyr 705), Cell Signaling \\
\hline Takemoto, S., et al. (2009) & $\mathrm{CC}$ & p-STAT3 & $\mathrm{IHC} \geq 5 \%$ & $\begin{array}{l}\text { anti-p-Stat3 (Tyr 705), sc-7993, Santa Cruz } \\
\text { Biotechnology }\end{array}$ \\
\hline \multirow[t]{2}{*}{ Tam, L., et al. (2007) } & $\mathrm{PC}$ & $\begin{array}{l}\text { p-STAT3- } \\
\text { cytoplasmic }\end{array}$ & $\mathrm{ICCC}>0.7$ & anti-p-STAT3 (Tyr 705), 9131, Cell Signaling \\
\hline & & p-STAT3-nuclear & & \\
\hline van Cruijsen, H., et al. (2009) & NSCLC & p-STAT3 & NR & anti-p-STAT3 (Tyr 705), clone D3A7, Cell Signaling \\
\hline Wang, M., et al. (2011) & NSCLC & p-STAT3 & $\mathrm{IHC}>25 \%$ & anti-p-STAT3, Cell Signaling Technology \\
\hline Wang, Y., et al. (2011) & Gliomas & p-STAT3 & IHC score $>4$ & anti-p-STAT3 (Tyr 705), clone D3A7, Cell Signaling \\
\hline Wang, Y. C., et al. (2011) & Osteosarcoma & STAT3 & $\mathrm{IHC}>5 \%$ & anti-STAT3, Santa Cruz Biotechnology \\
\hline Wu, Zheng-Sheng., et al. (2011) & Melanoma & p-STAT3 & NR & anti-p-STAT3, Santa Cruz Biotechnology \\
\hline Woo, S., et al. (2011) & $\mathrm{GC}$ & p-STAT3 & $\mathrm{IHC} \geq 1 \%$ & anti-p-STAT3, (Tyr 705), Cell Signaling \\
\hline \multirow[t]{2}{*}{ Xiong, Hua., et al. (2012) } & $\mathrm{GC}$ & STAT3 & $\mathrm{IHC}>15 \%$ & anti-STAT3 \\
\hline & & p-STAT3 & & anti-p-STAT3 (Tyr 705) \\
\hline Yakata, Yuichi., et al. (2007) & $\mathrm{GC}$ & p-STAT3 & $\mathrm{IHC}>10 \%$ & anti-p-STAT3, Santa Cruz Biotechnology \\
\hline Yamashita, H., et al. (2006) & $\mathrm{BC}$ & STAT3 & IHC score $\geq 2$ & anti-STAT3, (F-2), Santa Cruz Biotechnology \\
\hline Yang, C., et al. (2013) & $\mathrm{OC}$ & p-STAT3 & $\mathrm{IHC}>50 \%$ & anti-p-STAT3, (Tyr 705), Cell Signaling Technology \\
\hline Yang, Cao., et al. (2009) & Chordoma & p-STAT3 & IHC score $\geq 4$ & anti-p-STAT3, Cell Signaling Technology \\
\hline Yin, Z., et al. (2012) & NSCLC & STAT3 & $\mathrm{IHC} \geq 50 \%$ & anti-STAT3, (sc-8019); Santa Cruz \\
\hline You, Z., et al. (2012) & $\mathrm{EC}$ & p-STAT3 & IHC score $\geq 2$ & anti-p-STAT3, (Tyr 705), Cell Signaling Technology \\
\hline Yu, Y., et al. (2015) & NSCLC & pSTAT3 & IHC score $\geq 3$ & NR \\
\hline \multirow[t]{2}{*}{ Zhang, C. H., et al. (2012) } & $\mathrm{HC}$ & STAT3 & $\mathrm{IHC}>10 \%$ & anti-STAT3, Santa Cruz Biotechnology \\
\hline & & p-STAT3 & & anti-p-STAT3, (Tyr 705), Cell Signaling Technology \\
\hline Zhang, L. J., et al. (2013) & Wilms' tumor & STAT3 & $\mathrm{IHC}>51 \%$ & anti-STAT3, (sc-7179), Santa Cruz Biotechnology \\
\hline \multirow[t]{2}{*}{ Zhao, X., et al. (2012) } & SCLC & STAT3 & $\mathrm{IHC} \geq 25 \%$ & anti-STAT3, Wuhan Boster Company \\
\hline & & p-STAT3 & & anti-p-STAT3, clone B-7, Wuhan Boster Company \\
\hline Zhao, Yan., et al. (2012) & LSCC & STAT3 & $\mathrm{IHC} \geq 10 \%$ & anti-STAT3, Santa Cruz Biotechnology \\
\hline
\end{tabular}

NSCLC: Non-Small Cell Lung Cancer; GC: Gastric cancer; CRC: Colorectal Cancer; BC: Breast cancer; CC: Cervical Carcinoma; OC: Ovarian Carcinoma; PDA: Pancreatic Ductal Adenocarcinoma; TET: Thymic Epithelial Tumours; RCC: Renal Cell Carcinoma; HC: Hepatocellular Carcinoma; HNSCC: Head and Neck Squamous Cell Carcinoma; EC: Esophageal Cancer; OSCC: Oral Squamous Cell Carcinoma; SCLC: Small Cell Lung Cancer; NC: Nasopharyngeal carcinoma; LSCC: Lingual Squamous Cell Carcinoma; PC: Prostate Cancer; ICCH: Interclass Correlation Coefficient; NR: Not Reported. 
$\begin{array}{llll}\text { STAT3- } & \text { STAT3+ Odds Ratio Odds Ratio }\end{array}$

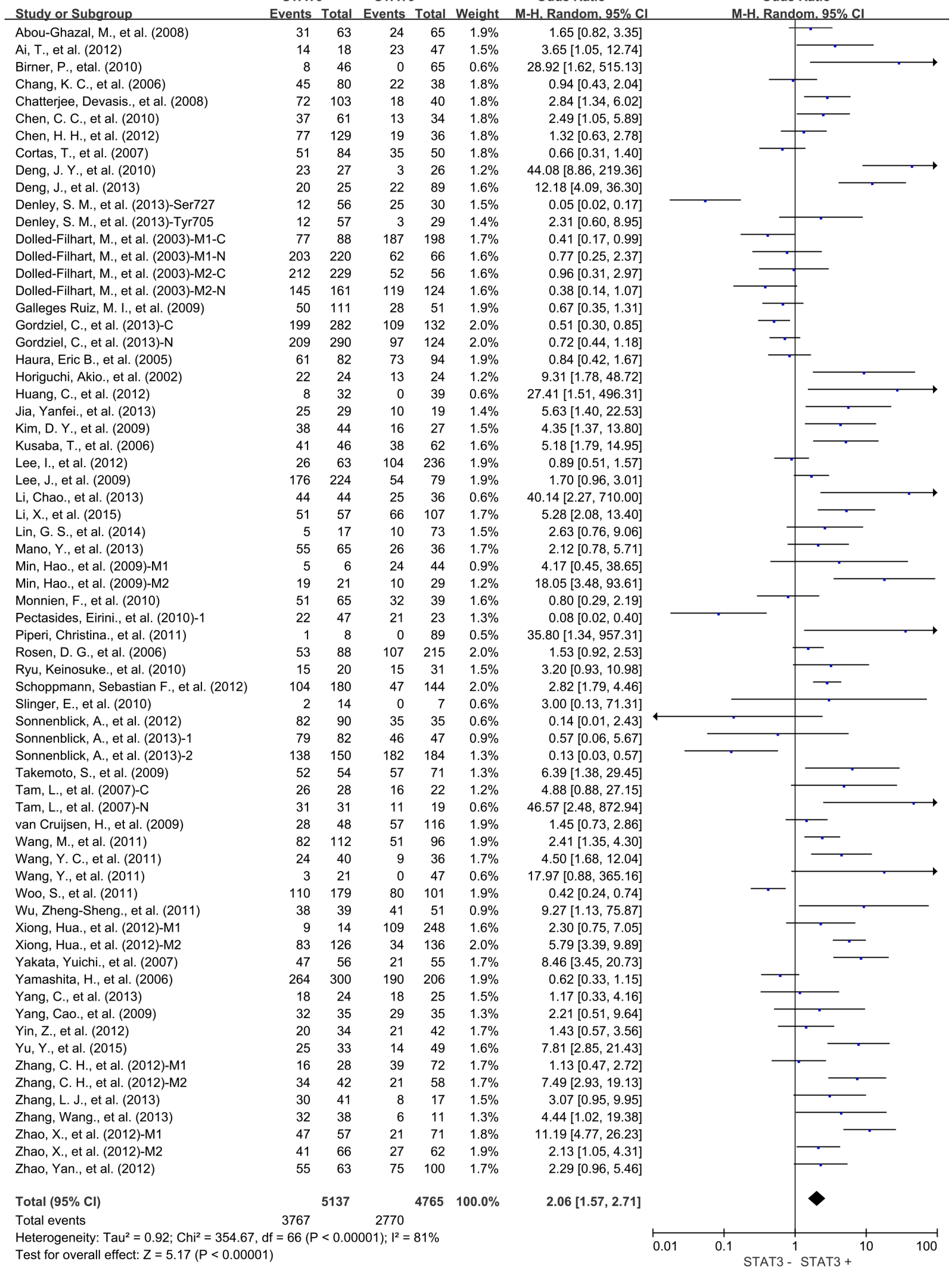

Figure 2: Three-year overall survival (OS) by STAT3 expression. M1: Marker 1, STAT3; M2: Marker 2, p-STAT3; 1: Cohort 1; 2: Cohort 2; N: nuclear expression; C: cytoplasmic expression. 


\section{A. Gastric cancer}

\begin{tabular}{|c|c|c|c|c|c|}
\hline Study or Subgroup & $\begin{array}{r}\text { STAT3 } \\
\text { Events }\end{array}$ & 3- & $\begin{array}{l}\text { STAT3 } \\
\text { Events }\end{array}$ & & \\
\hline Chatterjee, Devasis., et al. (2008) & 72 & 103 & 18 & 40 & $10.7 \%$ \\
\hline Deng, J. Y., et al. (2010) & 23 & 27 & 3 & 26 & $7.9 \%$ \\
\hline Deng, J., et al. (2013) & 20 & 25 & 22 & 89 & $9.7 \%$ \\
\hline Jia, Yanfei., et al. (2013) & 25 & 29 & 10 & 19 & $8.7 \%$ \\
\hline Kim, D. Y., et al. (2009) & 38 & 44 & 16 & 27 & $9.5 \%$ \\
\hline Lee, J., et al. (2009) & 176 & 224 & 54 & 79 & $11.2 \%$ \\
\hline Woo, S., et al. (2011) & 110 & 179 & 80 & 101 & $11.2 \%$ \\
\hline Xiong, Hua., et al. (2012)-M1 & 9 & 14 & 109 & 248 & $9.6 \%$ \\
\hline Xiong, Hua., et al. (2012)-M2 & 83 & 126 & 34 & 136 & $11.3 \%$ \\
\hline Yakata, Yuichi., et al. (2007) & 47 & 56 & 21 & 55 & $10.3 \%$ \\
\hline Total $(95 \% \mathrm{Cl})$ & & 827 & & 820 & $100.0 \%$ \\
\hline Total events & 603 & & 367 & & \\
\hline
\end{tabular}

Odds Ratio

Random. 95\% Cl $2.84[1.34,6.02]$ $44.08[8.86,219.36$ $12.18[4.09,36.30]$ $5.63[1.40,22.53]$ $4.35[1.37,13.80]$ $1.70[0.96,3.01]$ $0.42[0.24,0.74]$ $2.30[0.75,7.05]$ $5.79[3.39,9.89]$ $8.46[3.45,20.73]$

$4.06[1.86,8.89]$

\section{B. Lung cancer}

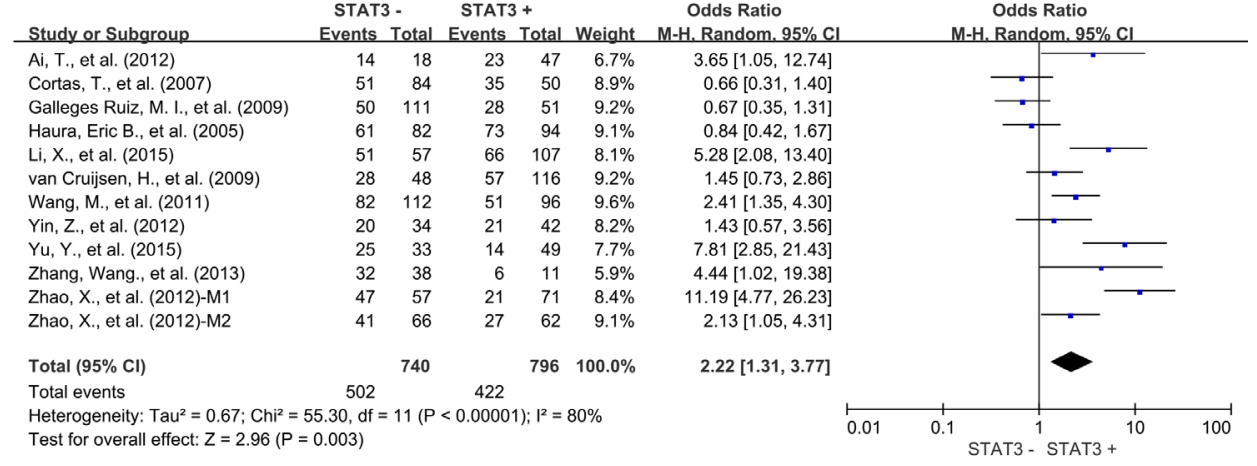

\section{Gliomas}

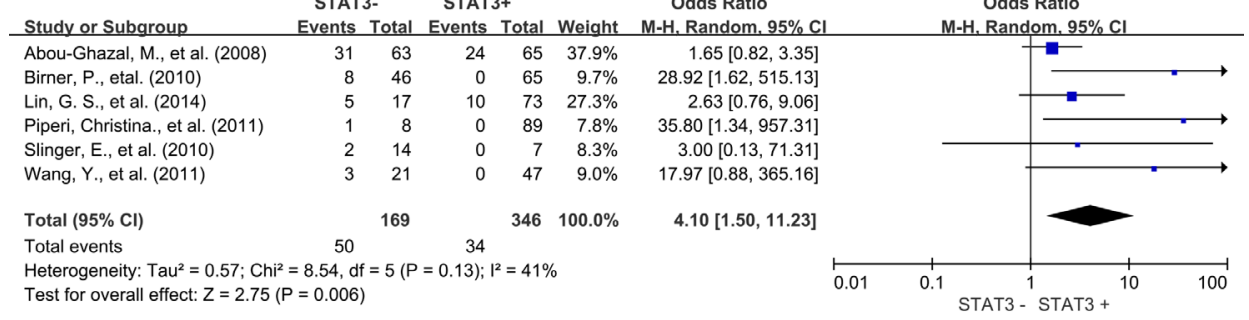

\section{Hepatocellular carcinoma}

STAT3 - STAT3 + Odds Ratio Odds Ratio

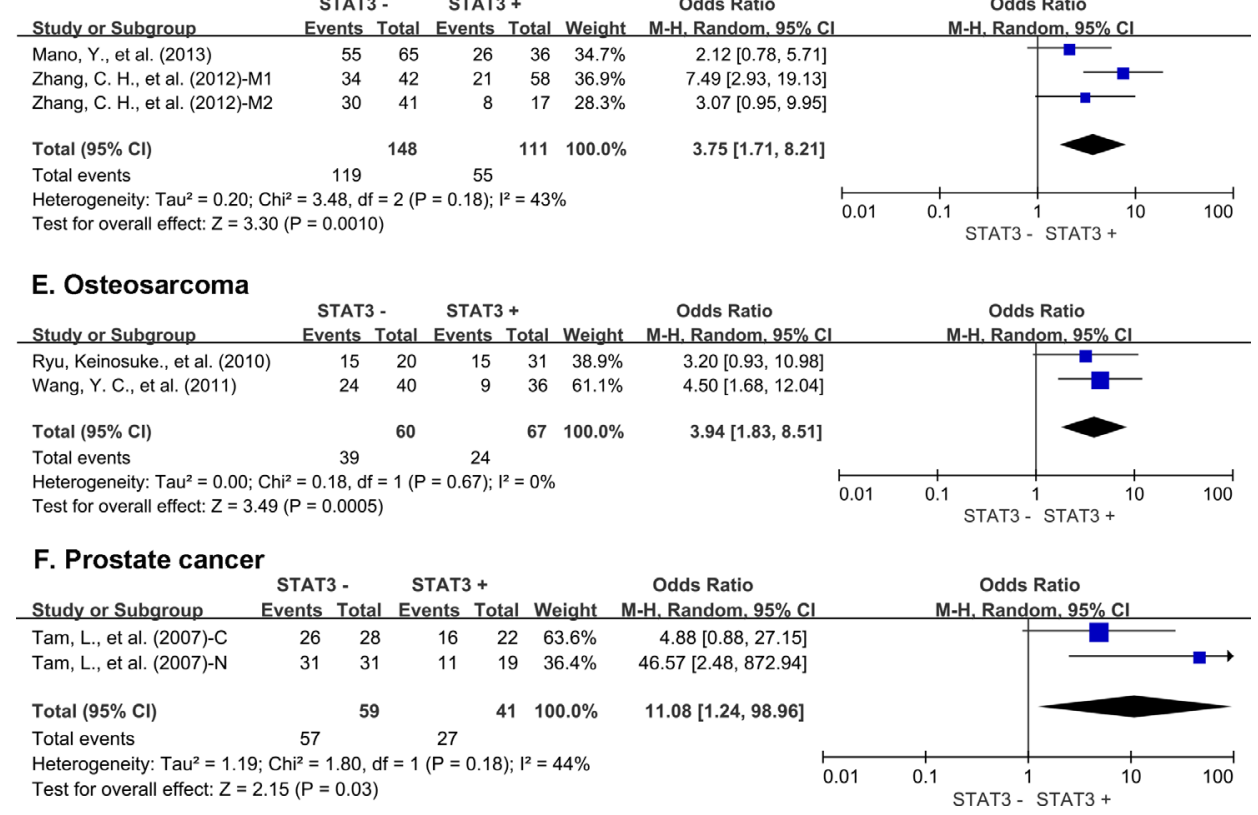

Figure 3: Subgroup analysis of 3-year OS by STAT3 expression in different tumor types. (A) gastric cancer; (B) lung cancer; (C) gliomas; (D) hepatic cancer; (E) osteosarcoma; (F) prostate cancer. M1: Marker 1, STAT3; M2: Marker 2, p-STAT3; 1: Cohort 1; 2: Cohort $2 \mathrm{~N}$ : nuclear expression; C: cytoplasmic expression. 
STAT3- $\quad$ STAT3+

Odds Ratio

Study or Subgroup

Events Total Events Total Weight M-H, Random, 95\% C

Abou-Ghazal, M., et al. (2008)

Chang, K. C., et al. (2006)

Chatterjee, Devasis., et al. (2008)

Chen, C. C., et al. (2010)

Chen, H. H., et al. (2012)

Cortas, T., et al. (2007)

Deng, J. Y., et al. (2010)

Deng, J., et al. (2013)

Denley, S. M., et al. (2013)-Ser727

Denley, S. M., et al. (2013)-Tyr705

Dolled-Filhart, M., et al. (2003)-M1-C

Dolled-Filhart, M., et al. (2003)-M1-N

Dolled-Filhart, M., et al. (2003)-M2-C

Dolled-Filhart, M., et al. (2003)-M2-N

Galleges Ruiz, M. I., et al. (2009)

Gordziel, C., et al. (2013)-C

Gordziel, C., et al. (2013)-N

Haura, Eric B., et al. (2005)

Hbibi, A. Tadlaoui., et al. (2008)-M1

Hbibi, A. Tadlaoui., et al. (2008)-M2

Horiguchi, Akio., et al. (2002)

Huang, C., et al. (2012)

Jia, Yanfei., et al. (2013)

Kim, D. Y., et al. (2009)

Kim, Yeon-Joo., et al. (2011)

Kusaba, T., et al. (2006)

Lee, I., et al. (2012)

Lee, J., et al. (2009)

Li, Chao., et al. (2013)

Mano, Y., et al. (2013)

Min, Hao., et al. (2009)-M1

Min, Hao., et al. (2009)-M2

Monnien, F., et al. (2010)

Pectasides, Eirini., et al. (2010)-1

Rosen, D. G., et al. (2006)

Ryu, Keinosuke., et al. (2010)

Schoppmann, Sebastian F., et al. (2012)

Sheen-Chen, Shyr-Ming., et al. (2008)

Sonnenblick, A., et al. (2012)

Sonnenblick, A., et al. (2013)-1

Sonnenblick, A., et al. (2013)-2

Takemoto, S., et al. (2009)

Tam, L., et al. (2007)-C

Tam, L., et al. (2007)-N

van Cruijsen, H., et al. (2009)

Wang, M., et al. (2011)

Wang, Y. C., et al. (2011)

Woo, S., et al. (2011)

Wu, Zheng-Sheng., et al. (2011)

Xiong, Hua., et al. (2012)-M1

Xiong, Hua., et al. (2012)-M2

Yakata, Yuichi., et al. (2007)

Yamashita, H., et al. (2006)

Yang, C., et al. (2013)

Yang, Cao., et al. (2009)

Yin, Z., et al. (2012)

Yu, Y., et al. (2015)

Zhang, C. H., et al. (2012)-M1

Zhang, C. H., et al. (2012)-M2

Zhang, L. J., et al. (2013)

Zhang, Wang., et al. (2013)

Zhao, X., et al. (2012)-M1

Zhao, X., et al. (2012)-M2

Zhao, Yan., et al. (2012)

Total $(95 \% \mathrm{CI})$

Total events

$\begin{array}{rrrr}26 & 63 & 23 & 65 \\ 24 & 80 & 13 & 38 \\ 63 & 103 & 0 & 40 \\ 32 & 61 & 11 & 34\end{array}$

$1.9 \%$

$1.28[0.63,2.62]$

$0.82[0.36,1.88]$

$127.00[7.60,2123.38]$

$2.31[0.96,5.54]$

$1.07[0.51,2.24]$

$0.86[0.43,1.75]$

$143.75[14.95,1382.11]$

$9.63[3.39,27.36]$

$7.85[0.43,144.32]$

$7.45[0.40,136.95]$

$0.67[0.35,1.28]$

$0.36[0.14,0.95]$

$0.74[0.31,1.76]$

$0.36[0.18,0.75]$

$0.61[0.31,1.19]$

$0.54[0.34,0.86]$

$0.67[0.42,1.05]$

$0.82[0.45,1.48]$

$0.88[0.36,2.16]$

$0.63[0.28,1.43]$

$9.31[1.78,48.72]$

$15.80[0.84,297.59]$

$7.93[1.54,40.74]$

$6.55[2.21,19.36]$

$5.50[1.16,26.02]$

$8.75[3.05,25.09]$

$1.18[0.62,2.24]$

$1.63[0.96,2.78]$

$48.06[5.96,387.70]$

$3.50[1.35,9.05]$

0.18 [0.01, 3.42]

$77.63[4.19,1439.00]$

$0.77[0.32,1.83]$

$0.22[0.07,0.66]$

$2.02[1.22,3.36]$

$5.45[1.56,19.06]$

$3.35[2.08,5.40]$

$2.35[0.92,6.00]$

$0.20[0.04,0.90]$

$1.05[0.24,4.61]$

$0.25[0.12,0.53]$

$4.55[1.25,16.65]$

$4.15[1.07,16.14]$

19.94 [3.65, 108.90]

$2.05[1.03,4.08]$

$1.94[1.07,3.53]$

$4.50[1.68,12.04]$

$0.40[0.23,0.68]$

3.21 [1.27, 8.09]

$1.91[0.64,5.70]$

$6.82[3.64,12.78]$

$5.94[2.57,13.73]$

$0.73[0.45,1.19]$

$2.98[0.93,9.57]$

$3.69[0.91,15.05]$

$1.90[0.76,4.76]$

$3.67[1.39,9.72]$

2.94 [1.04, 8.28]

$1.52[0.59,3.92]$

3.07 [0.95, 9.95]

$2.31[0.59,9.02]$

$18.05[0.99,327.58]$

$6.89[0.35,136.15]$

$5.31[2.56,11.00]$

$2.00[1.53,2.63]$

$4456 \quad 100.0 \%$ 3221 2124

Heterogeneity: $\mathrm{Tau}^{2}=0.87 ; \mathrm{Chi}^{2}=354.57, \mathrm{df}=63(\mathrm{P}<0.00001) ; \mathrm{I}^{2}=82 \%$

Test for overall effect: $Z=5.03(P<0.00001)$

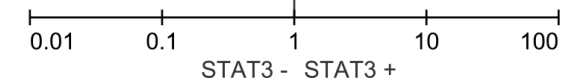

Figure 4: Five-year OS by STAT3 expression. M1: Marker 1, STAT3; M2: Marker 2, p-STAT3; 1: Cohort 1; 2: Cohort 2 N: nuclear expression; C: cytoplasmic expression. 


\section{A. Gastric cancer}

Study or Subgroup

Chatterjee, Devasis., et al. (2008)

STAT3 - $\quad$ STAT3 +

Odds Ratio

Events Total Events Total Weight M-H, Random, 95\% Cl

Deng, J. Y., et al. (2010)

Deng, J., et al. (2013)

Jia, Yanfei., et al. (2013)

Kim, D. Y., et al. (2009)

Lee, J., et al. (2009)

Woo, S., et al. (2011)

Xiong, Hua., et al. (2012)-M1

Xiong, Hua., et al. (2012)-M2

Yakata, Yuichi., et al. (2007)

$\begin{array}{rrrrrr}63 & 103 & 0 & 40 & 5.8 \% & 127.00[7.60,2123.38]\end{array}$

Total $(95 \% \mathrm{CI})$

$\begin{array}{rrrrrr}63 & 103 & 0 & 40 & 5.8 \% & 127.00[7.60,2123.38] \\ 23 & 27 & 1 & 26 & 7.2 \% & 143.75[14.95,1382.11]\end{array}$

$\begin{array}{llllll}13 & 25 & 9 & 89 & 10.6 \% & 9.63[3.39,27.36]\end{array}$

$\begin{array}{llllll}14 & 29 & 2 & 19 & 8.9 \% & 7.93[1.54,40.74]\end{array}$

$\begin{array}{llllll}36 & 44 & 11 & 27 & 10.5 \% & 6.55[2.21,19.36]\end{array}$

$\begin{array}{lllll}158 & 224 & 47 & 79 & 11.8 \%\end{array}$

$\begin{array}{lllll}98 & 179 & 76 & 101 & 11.8 \%\end{array}$

$1.63[0.96,2.78]$

$0.40[0.23,0.68]$

$1.91[0.64,5.70]$

$6.82[3.64,12.78]$

$\begin{array}{lllll}60 & 126 & 16 & 136 & 11.6 \%\end{array}$

$5.94[2.57,13.73]$

$5.48[2.14,14.01]$

Total events

$515 \quad 253$

Heterogeneity: Tau $^{2}=1.87 ; \mathrm{Chi}^{2}=93.33, \mathrm{df}=9(\mathrm{P}<0.00001) ; \mathrm{I}^{2}=90 \%$

Test for overall effect: $Z=3.55(P=0.0004)$

$820 \quad 100.0 \%$

\section{B. Hepatocellular carcinoma \\ STAT3 -}

STAT3 +

Random, 95\% C

Study or Subgroup

Events Total Events Total Weight M-H. Random. 95\% C

Mano, Y., et al. (2013)

Zhang, C. H., et al. (2012)-M1

$\begin{array}{lllll}55 & 65 & 22 & 36 & 35.2 \%\end{array}$

$3.50[1.35,9.05]$

Zhang, C. H., et al. (2012)-M2

$\begin{array}{lllll}9 & 28 & 10 & 72 & 29.6 \%\end{array}$

$2.94[1.04,8.28]$

$1.52[0.59,3.92]$

Total $(95 \% \mathrm{CI})$

$\begin{array}{lllll}11 & 42 & 11 & 58 & 35.2 \%\end{array}$

$2.48[1.41,4.35]$

Total events

$75 \quad 135$

Heterogeneity: Tau$^{2}=0.00 ; \mathrm{Chi}^{2}=1.64, \mathrm{df}=2(\mathrm{P}=0.44) ; \mathrm{I}^{2}=0 \%$

Test for overall effect: $Z=3.15(P=0.002)$

$166 \quad 100.0 \%$

\section{Osteosarcoma}

STAT3 - $\quad$ STAT3 +

Odds Ratio

Events Total Events Total Weight M-H, Random, 95\% Cl

5.45 [1.56, 19.06]

$4.50[1.68,12.04]$

Ryu, Keinosuke., et al. (2010)

$\begin{array}{rrrrr}15 & 20 & 11 & 31 & 38.2 \% \\ 24 & 40 & 9 & 36 & 61.8 \%\end{array}$

Total $(95 \% \mathrm{CI})$

$60 \quad 67 \quad 100.0 \%$

$4.84[2.23,10.50]$

Total events

39 20

Heterogeneity: $\mathrm{Tau}^{2}=0.00 ; \mathrm{Chi}^{2}=0.06, \mathrm{df}=1(\mathrm{P}=0.81) ; \mathrm{I}^{2}=0 \%$

Test for overall effect: $Z=4.00(P<0.0001)$

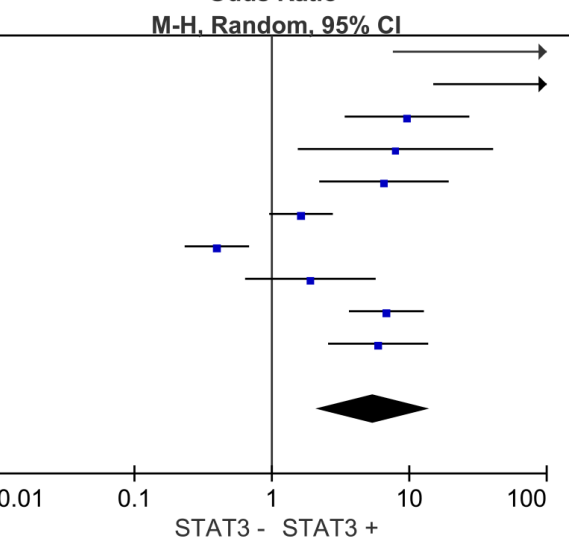

\section{Pancreatic cancer}

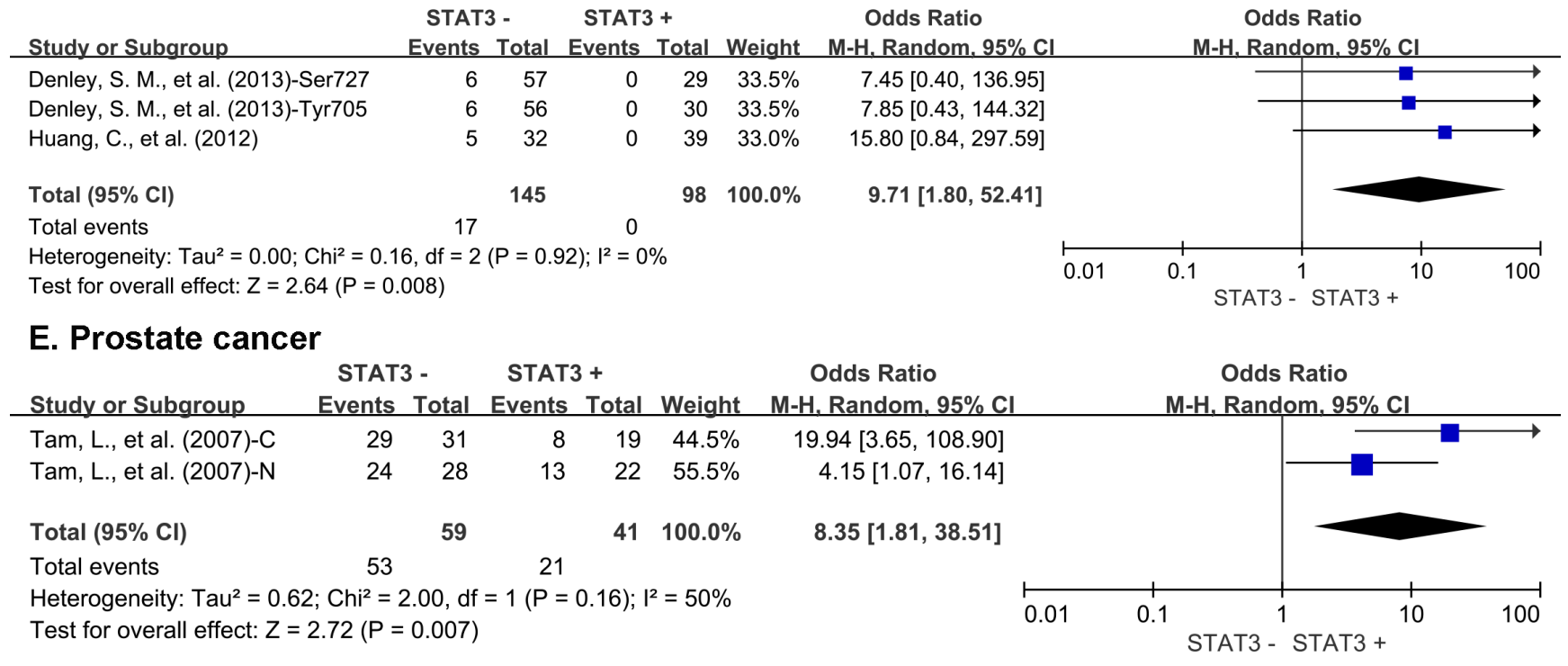

Figure 5: Subgroup analysis of 5-year OS by STAT3 expression in different tumor types. (A) gastric cancer; (B) hepatic cancer; (C) osteosarcoma; (D) pancreatic cancer; (E) prostate cancer. M1: Marker 1, STAT3; M2: Marker 2, p-STAT3; 1: Cohort 1; 2: Cohort $2 \mathrm{~N}$ : nuclear expression; C: cytoplasmic expression. 


\section{Association of STAT3 with DFS}

Meta-analysis of 10 studies showed that STAT3 expression was associated with statistically significant poor 3-year DFS $(\mathrm{OR}=3.52,95 \% \mathrm{CI}=1.85$ to $6.71, P=0.0001$ ) (Figure 8A) and poor 5-year DFS $(\mathrm{OR}=3.37,95 \% \mathrm{CI}=1.67$ to $6.80, P=0.0007)$ (Figure 8B).

\section{Sensitivity analyses}

Removal of the studies that was an outlier (score, IRS, $>50 \%$ vs $1 \%-6 \%$ for other studies) or no report (NR) with regard to the cutoff of STAT3 overexpression by IHC did not influence results for 3 - or 5-year $\mathrm{OS}(\mathrm{OR}=2.45,95 \% \mathrm{CI}=1.73$ to 3.48 , $p<0.00001 ; \mathrm{OR}=2.08,95 \% \mathrm{CI}=1.46$ to 2.96 , $p<0.0001$; respectively). Exclusion of these studies did not reduce heterogeneity for 3- or 5-year OS (Cochran's Q $P<0.00001, \mathrm{I}^{2}=82 \%$; Cochran's Q $P<0.00001$, $\mathrm{I}^{2}=84 \%$, respectively).

Removal of studies with NOS score 6 did not influence results for 3- or 5-year OS (OR $=2.49,95 \%$ $\mathrm{CI}=1.86$ to $3.34, p<0.00001 ; \mathrm{OR}=2.33,95 \%$
$\mathrm{CI}=1.74$ to $3.12, p<0.00001$, respectively). Exclusion of these studies did not reduce heterogeneity for 3- or 5-year OS (Cochran's Q $P<0.00001, \mathrm{I}^{2}=80 \%$; Cochran's Q $P<0.00001, \mathrm{I}^{2}=82 \%$, respectively).

\section{Publication bias}

Funnel plot analysis showed that there was no statistical evidence of publication bias in our meta-analysis (data not shown).

\section{DISCUSSION}

This meta-analysis is the most comprehensive assessment of the literatures regarding STAT3 expression and tumor prognosis to date. We systematically evaluated survival data for 9449 solid tumor patients included in 63 different studies. Our study demonstrated that the expression of STAT3 is a marker of poor prognosis in solid tumors, with consistent results of OS at 3 and 5 years. Regarding to the tumor types, elevated STAT3 expression in tumor tissues were associated with worse OS of gastric cancer, lung cancer, gliomas, hepatic cancer, osteosarcoma, prostate cancer and pancreatic cancer.

\begin{tabular}{|c|c|c|c|c|c|c|c|c|c|c|}
\hline Study or Subgroup & $\begin{array}{l}\text { TNM stage } \\
\text { Events }\end{array}$ & $\begin{array}{l}\text { I }- \text { II } \\
\text { Total }\end{array}$ & \multicolumn{2}{|c|}{ TNM stage III-IV } & \multicolumn{2}{|r|}{ Odds Ratio } & \multicolumn{3}{|c|}{$\begin{array}{c}\text { Odds Ratio } \\
\text { M-H, Random, } 95 \% \mathrm{Cl}\end{array}$} & \\
\hline Ai, T., et al. (2012) & 36 & 46 & 11 & 19 & $3.5 \%$ & $2.62[0.83,8.26]$ & & & & \\
\hline Chang, K. C., et al. (2006) & 0 & 1 & 14 & 39 & $0.8 \%$ & $0.59[0.02,15.35]$ & & & & \\
\hline Chen, H. H., et al. (2012) & 27 & 124 & 9 & 41 & $4.5 \%$ & $0.99[0.42,2.32]$ & & & & \\
\hline Cortas, T., et al. (2007) & 4 & 19 & 19 & 75 & $3.3 \%$ & $0.79[0.23,2.66]$ & & & & \\
\hline Dobi, E., et al. (2013) & 34 & 66 & 5 & 5 & $1.0 \%$ & $0.10[0.01,1.82]$ & & & & \\
\hline Huang, C., et al. (2012) & 5 & 22 & 14 & 26 & $3.2 \%$ & $0.25[0.07,0.89]$ & & & & \\
\hline Jia, Yanfei., et al. (2013) & 11 & 43 & 16 & 28 & $4.0 \%$ & $0.26[0.09,0.71]$ & & & & \\
\hline Kim, D. Y., et al. (2009) & 44 & 70 & 18 & 38 & $4.7 \%$ & $1.88[0.84,4.19]$ & & & & \\
\hline Kusaba, T., et al. (2006) & 16 & 47 & 20 & 33 & $4.3 \%$ & $0.34[0.13,0.84]$ & & & & \\
\hline Li, Chao., et al. (2013) & 42 & 76 & 65 & 88 & $5.2 \%$ & $0.44[0.23,0.84]$ & & & & \\
\hline Li, X., et al. (2015) & 9 & 18 & 25 & 32 & $3.3 \%$ & $0.28[0.08,0.97]$ & & & & \\
\hline Min, Hao., et al. (2009)-M1 & 5 & 18 & 22 & 32 & $3.2 \%$ & $0.17[0.05,0.62]$ & & & & \\
\hline Min, Hao., et al. (2009)-M2 & 32 & 56 & 151 & 247 & $5.5 \%$ & $0.85[0.47,1.53]$ & & & & \\
\hline Rosen, D. G., et al. (2006) & 29 & 65 & 55 & 70 & $4.9 \%$ & $0.22[0.10,0.47]$ & & & & \\
\hline Shah, N. G., et al. (2006) & 85 & 93 & 163 & 169 & $3.7 \%$ & $0.39[0.13,1.16]$ & & & & \\
\hline Xiong, Hua., et al. (2012)-M1 & 38 & 93 & 98 & 169 & $5.8 \%$ & $0.50[0.30,0.84]$ & & & & \\
\hline Xiong, Hua., et al. (2012)-M2 & 17 & 40 & 25 & 36 & $4.2 \%$ & $0.33[0.13,0.84]$ & & & & \\
\hline Yin, Z., et al. (2012) & 16 & 38 & 33 & 44 & $4.2 \%$ & $0.24[0.09,0.62]$ & & & & \\
\hline Yu, Y., et al. (2015) & 43 & 60 & 29 & 40 & $4.4 \%$ & $0.96[0.39,2.34]$ & & & & \\
\hline Zhang, C. H., et al. (2012)-M1 & 35 & 60 & 23 & 40 & $4.7 \%$ & $1.03[0.46,2.33]$ & & & & \\
\hline Zhang, C. H., et al. (2012)-M2 & 10 & 44 & 7 & 14 & $3.2 \%$ & $0.29[0.08,1.04]$ & & & & \\
\hline Zhang, L. J., et al. (2013) & 29 & 77 & 44 & 51 & $4.3 \%$ & $0.10[0.04,0.24]$ & & & & \\
\hline Zhao, X., et al. (2012)-M1 & 26 & 77 & 36 & 51 & $4.8 \%$ & $0.21[0.10,0.46]$ & & & & \\
\hline Zhao, X., et al. (2012)-M2 & 59 & 112 & 41 & 51 & $4.8 \%$ & $0.27[0.12,0.59]$ & & & & \\
\hline Zhao, Yan., et al. (2012) & 59 & 112 & 41 & 51 & $4.8 \%$ & $0.27[0.12,0.59]$ & & & & \\
\hline Total $(95 \% \mathrm{Cl})$ & & 1477 & & 1489 & $100.0 \%$ & $0.42[0.31,0.58]$ & & & & \\
\hline Total events & 711 & & 984 & & & & & & & \\
\hline $\begin{array}{l}\text { Heterogeneity: } \mathrm{Tau}^{2}=0.37 ; \mathrm{Ch} \\
\text { Test for overall effect: } \mathrm{Z}=5.42\end{array}$ & $\begin{array}{l}=66.72, \mathrm{df}= \\
<0.00001)\end{array}$ & $24(P$ & $.00001)$ & $64 \%$ & & & 0.01 & $\begin{array}{cc}0.1 & 1 \\
\text { TNM stage } & \text { I - II }\end{array}$ & $\begin{array}{l}10 \\
\text { TNM stage I }\end{array}$ & III-IV \\
\hline
\end{tabular}

Figure 6: Subgroup analysis the correlation of STAT3 expression and tumor stage. M1: Marker 1, STAT3; M2: Marker 2, p-STAT3. 
STAT3 - $\quad$ STAT3 + Odds Ratio Odds Ratio

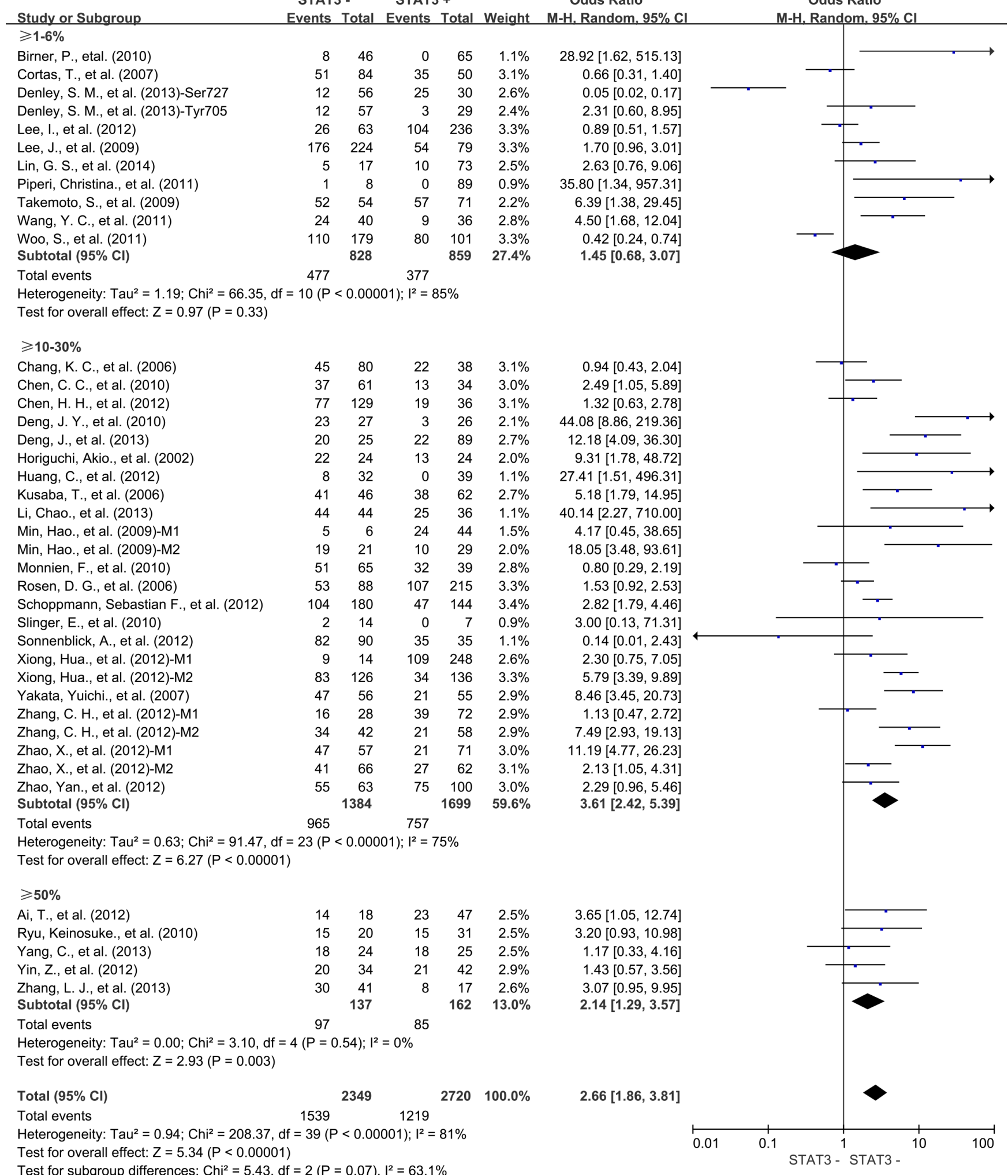

Figure 7: Subgroup analysis the correlation between STAT3 overexpression and 3-year OS of solid tumors according to cut-off values determining STAT3 positivity. M1: Marker 1, STAT3; M2: Marker 2, p-STAT3. 
However, elevated STAT3 expression was associated with better prognosis of breast cancer. In addition, expression level of phosphorylated STAT3 was more significantly associated with worse outcome of solid tumors than unphosphorylated STAT3.

Our study found there is no significant correlation between STAT3 overexpression and OS of colorectal cancer and ovarian cancer. And STAT3 overexpression in breast cancer tissue is associated with favorable OS. However, recent studies demonstrated that STAT3targeted inhibitor could restrain tumor development in various solid tumor models including breast cancer [16, 19, 85, 86], melanoma [87] and ovarian cancer $[16,88]$. These divergences suggest that further study is needed to shed more light on the underling mechanism of STAT3 signal pathway in pro-tumor microenvironment in different tumor types.

There are several important implications in this meta-analysis. First, it shows that STAT3 expression is related to adverse outcome of most solid tumors. Second, it identifies a subgroup of tumors with unfavorable outcome in gastric cancer, lung cancer, hepatic cancer, prostate cancer and glioblastoma, but with favorable outcome in breast cancer. Finally, it emphasizes the potential of STAT3 to developing a valuable therapeutic target and prognostic biomarker for solid tumor.

This study also has some limitations. First, from the literature we could only extract summarized population-level data rather than individual patient-level data. Second, the method for assessing STAT3 expression and definition of STAT3 positivity are inconsistent. Finally, substantial heterogeneity observed across included studies cannot be fully accounted for by our use of appropriate meta-analytic techniques with randomeffects modeling.

In summary, STAT3 expression in solid tumor tissues is associated with poor survival in most solid tumors, which suggests that STAT3 is a valuable prognostic biomarker and a promising therapeutic target for solid tumors.

\begin{tabular}{|c|c|c|c|c|c|}
\hline Study or Subgroup & $\begin{array}{l}\text { STAT } \\
\text { Events }\end{array}$ & Total & $\begin{array}{l}\text { STAT3 } \\
\text { Events }\end{array}$ & & Weight \\
\hline Choi, Chel Hun., et al. (2010) & 8 & 9 & 10 & 20 & $5.1 \%$ \\
\hline Lee, J., et al. (2009) & 165 & 224 & 49 & 79 & $12.5 \%$ \\
\hline Li, X., et al. (2015) & 50 & 57 & 61 & 107 & $10.9 \%$ \\
\hline Macha, Muzafar A., et al. (2011) & 17 & 31 & 12 & 63 & $10.6 \%$ \\
\hline Mano, Y., et al. (2013) & 36 & 65 & 5 & 36 & $10.0 \%$ \\
\hline Schoppmann, Sebastian F., et al. (2012) & 87 & 180 & 37 & 144 & $12.8 \%$ \\
\hline Takemoto, S., et al. (2009) & 53 & 54 & 58 & 71 & $5.7 \%$ \\
\hline Wang, Y. C., et al. (2011) & 27 & 40 & 12 & 36 & $10.5 \%$ \\
\hline Yamashita, H., et al. (2006) & 224 & 300 & 171 & 206 & $12.9 \%$ \\
\hline Zhang, L. J., et al. (2013) & 30 & 41 & 5 & 17 & $9.0 \%$ \\
\hline Total $(95 \% \mathrm{Cl})$ & \multicolumn{3}{|c|}{1001} & 779 & $100.0 \%$ \\
\hline Total events & 697 & & 420 & & \\
\hline \multicolumn{6}{|c|}{$\begin{array}{l}\text { Heterogeneity: } \mathrm{Tau}^{2}=0.79 ; \mathrm{Chi}^{2}=54.34, \mathrm{df}=9(\mathrm{P}<0.00001) ; \mathrm{I}^{2}=83 \% \\
\text { Test for overall effect: } \mathrm{Z}=3.82(\mathrm{P}=0.0001)\end{array}$} \\
\hline
\end{tabular}

Odds Ratio Odds Ratio

H. Random. $95 \% \mathrm{Cl} \quad \mathrm{M}-\mathrm{H}$. Random. $95 \% \mathrm{Cl}$

$8.00[0.84,76.37]$

$1.71[0.99,2.95]$

$5.39[2.24,12.97]$

$5.16[2.00,13.30]$

$7.70[2.66,22.30]$

$2.71[1.68,4.35]$

$11.88[1.50,93.93]$

$4.15[1.59,10.83]$

$0.60[0.39,0.94]$

$6.55[1.87,22.87]$

$3.52[1.85,6.71]$

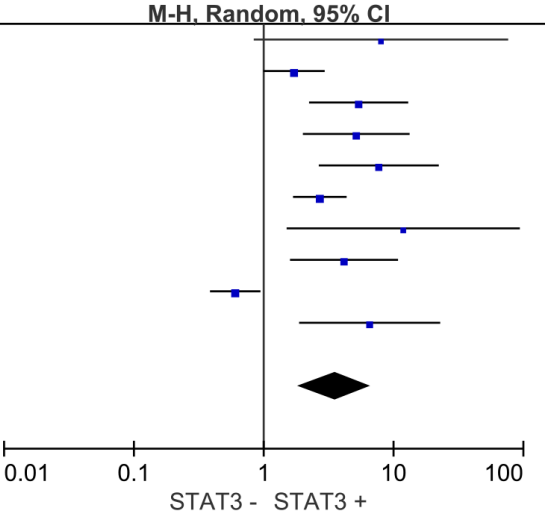

B

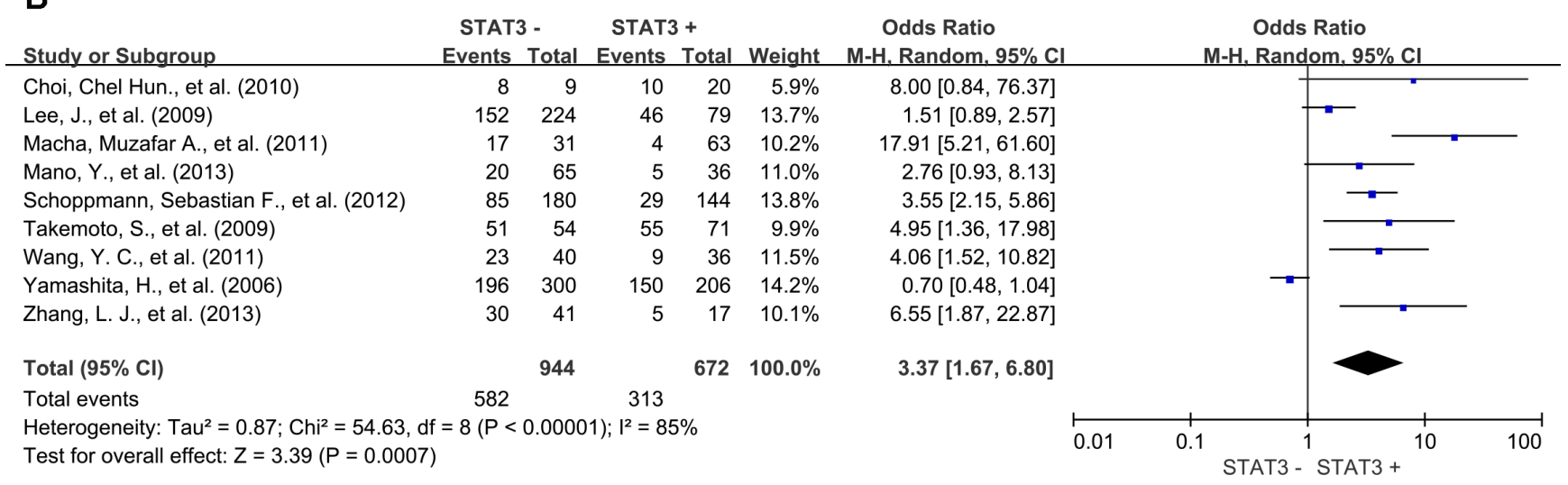

Figure 8: Three and five-year DFS by STAT3 expression. (A) 3-year DFS; (B) 5-year DFS. M1: Marker 1, STAT3; M2: Marker 2, p-STAT3. 


\section{MATERIALS AND METHODS}

This meta-analysis was conducted according to the statement for reporting systematic reviews and metaanalyses [89]. This study summarized and analyzed the results of previous studies, so the ethical approval was not necessary.

\section{Search strategy and study selection}

An electronic search of Pubmed, Web of Science and EBSCO were undertaken for studies evaluating STAT3 or p-STAT3 expression and clinical outcome in solid tumors from 1994 to August 2015. The search was performed with subject heading terms including "signal transducer and activator of transcription 3" or "STAT3 transcription factor" or "STAT3" or "phosphorylated signal transducer and activator of transcription 3" or "phosphorylated STAT3 transcription factor" or "phospho-STAT3" and "neoplasms" and the results were limited to human studies of solid tumors. In addition, the entry "signal transducer and activator of transcription 3" or "STAT3 transcription factor" or "STAT3" or "phosphorylated signal transducer and activator of transcription 3" or "phosphorylated STAT3 transcription factor" or "phospho-STAT3" and the name of each specific solid tumor were used for additional studies. A total of 3547, 3542 and 2914 entries were identified, respectively. Inclusion criteria were the measurement of STAT3 and (or) p-STAT3 by immunohistochemistry (IHC), availability of survival data for at least 3 years, and original article written in English. Exclusion criteria were studies evaluating gene expression of STAT3 measured by polymerase chain reaction (PCR) and STAT3 expression in lymph node and myeloid cells. Citation lists of retrieved articles were manually screened to ensure sensitivity of the search strategy. Study selection was based on the association of STAT3 and survival. Two reviewers (Pin Wu and Dang $\mathrm{Wu}$ ) evaluated independently all of the full articles for study eligibility. Inter-reviewer agreement was assessed using Cohen's kappa coefficient. Disagreement was resolved by consensus.

\section{Data extraction}

Overall survival (OS) and disease free survival (DFS) were the primary endpoints of interest. Data were extracted using predefined abstraction forms. The following details were extracted by two authors (Pin $\mathrm{Wu}$ and Dang $\mathrm{Wu}$ ): name of first author, year of publication, country of publication, tumor type, patient number, tumor stage, antibodies used for the evaluation, method and score for STAT3 assessment, and cut-off values to determine STAT3 positivity. Data for 3 and 5 year of OS and DFS were extracted from tables or Kaplan-Meier curves for both STAT3 negative and STAT3 positive group.
The studies included in our meta-analysis were all cohort studies. Two independent authors evaluated the quality of each included study using Newcastle-Ottawa Scale (NOS) [90]. The studies with 6 scores or more were considered as high quality studies. A consensus NOS score for each item was achieved finally.

\section{Data synthesis}

The relative frequency of OS and DFS at 3 and 5 years between STAT3 negative and STAT3 positive group was presented as an odds ratio (OR) and its $95 \%$ confidence interval (CI). Sensitivity analyses were carried out for different analytical methods and cut-offs for defining STAT3 expression and NOS scores for quality assessment of included studies. Publication bias was assessed by visual inspection of the funnel plot.

\section{Statistical analysis}

Data were extracted from the primary publications and combined into a meta-analysis using RevMan 5.3 analysis software (Cochrane Collaboration, Copenhagen, Denmark). Estimates of ORs were weighted and pooled using the Mantel-Haenszel random effect model. Statistical heterogeneity was assessed using the Cochran's $\mathrm{Q}$ and $\mathrm{I}^{2}$ statistics. Differences between subgroups were assessed using methods as previous described by Deeks et al [91]. Meta-regression analysis was conducted using Stata 12.0 software (StataCorp LP, College Station, TX). All statistical tests were two-sided, and statistical significance was defined as $P$ less than 0.05 . No correction was made for multiple statistical testing.

\section{ACKNOWLEDGMENTS AND FUNDING}

We thank all the group members for helpful discussions. This work was supported by grants from the National Natural Science Foundation of China (81572800, PW), the Science and Technology Department of Zhejiang Province (2013c03044-7, YC), and Natural Science Foundation of Zhejiang Province (LY13H160016, YC; Y15H160094, GS and LY15H160041, PW).

\section{CONFLICTS OF INTEREST}

The authors declare no competing financial interest.

\section{REFERENCES}

1. Yu H, Pardoll D, Jove R. STATs in cancer inflammation and immunity: a leading role for STAT3. Nat Rev Cancer. 2009; 9:798-809.

2. Ernst M, Najdovska M, Grail D, Lundgren-May T, Buchert M, Tye H, Matthews VB, Armes J, Bhathal PS, Hughes NR, Marcusson EG, Karras JG, Na S, et al. STAT3 
and STAT1 mediate IL-11-dependent and inflammationassociated gastric tumorigenesis in gp130 receptor mutant mice. J Clin Invest. 2008; 118:1727-1738.

3. Gao SP, Mark KG, Leslie K, Pao W, Motoi N, Gerald WL, Travis WD, Bornmann W, Veach D, Clarkson B, Bromberg JF. Mutations in the EGFR kinase domain mediate STAT3 activation via IL-6 production in human lung adenocarcinomas. J Clin Invest. 2007; 117:3846-3856.

4. Kortylewski M, Xin H, Kujawski M, Lee H, Liu Y, Harris T, Drake C, Pardoll D, Yu H. Regulation of the IL-23 and IL-12 balance by Stat 3 signaling in the tumor microenvironment. Cancer Cell. 2009; 15:114-123.

5. Crescenzo R, Abate F, Lasorsa E, Tabbo F, Gaudiano M, Chiesa N, Di Giacomo F, Spaccarotella E, Barbarossa L, Ercole E, Todaro M, Boi M, Acquaviva A, et al. Convergent mutations and kinase fusions lead to oncogenic STAT3 activation in anaplastic large cell lymphoma. Cancer Cell. 2015; 27:516-532.

6. Fan QW, Cheng CK, Gustafson WC, Charron E, Zipper P, Wong RA, Chen J, Lau J, Knobbe-Thomsen C, Weller M, Jura N, Reifenberger G, Shokat KM, et al. EGFR phosphorylates tumor-derived EGFRvIII driving STAT3/5 and progression in glioblastoma. Cancer Cell. 2013; 24:438-449.

7. Yu H, Lee H, Herrmann A, Buettner R, Jove R. Revisiting STAT3 signalling in cancer: new and unexpected biological functions. Nat Rev Cancer. 2014; 14:736-746.

8. Gough DJ, Corlett A, Schlessinger K, Wegrzyn J, Larner AC, Levy DE. Mitochondrial STAT3 supports Rasdependent oncogenic transformation. Science 2009; 324:1713-1716.

9. Lee HJ, Zhuang G, Cao Y, Du P, Kim HJ, Settleman J. Drug resistance via feedback activation of Stat3 in oncogeneaddicted cancer cells. Cancer Cell. 2014; 26:207-221.

10. Dai B, Meng J, Peyton M, Girard L, Bornmann WG, Ji L, Minna JD, Fang B, Roth JA. STAT3 mediates resistance to MEK inhibitor through microRNA miR-17. Cancer Res. 2011; 71:3658-3668.

11. Ernst M, Thiem S, Nguyen PM, Eissmann M, Putoczki TL. Epithelial gp130/Stat3 functions: an intestinal signaling node in health and disease. Semin Immunol. 2014; 26:29-37.

12. Guryanova $\mathrm{OA}, \mathrm{Wu} \mathrm{Q}$, Cheng L, Lathia JD, Huang Z, Yang J, MacSwords J, Eyler CE, McLendon RE, Heddleston JM, Shou W, Hambardzumyan D, Lee J, et al. Nonreceptor tyrosine kinase BMX maintains self-renewal and tumorigenic potential of glioblastoma stem cells by activating STAT3. Cancer Cell. 2011; 19:498-511.

13. Kim E, Kim M, Woo DH, Shin Y, Shin J, Chang N, Oh YT, Kim H, Rheey J, Nakano I, Lee C, Joo KM, Rich JN, et al. Phosphorylation of EZH2 activates STAT3 signaling via STAT3 methylation and promotes tumorigenicity of glioblastoma stem-like cells. Cancer Cell. 2013; 23:839-852.
14. Deng J, Liu Y, Lee H, Herrmann A, Zhang W, Zhang C, Shen S, Priceman SJ, Kujawski M, Pal SK, Raubitschek A, Hoon DS, Forman S, et al. S1PR1-STAT3 signaling is crucial for myeloid cell colonization at future metastatic sites. Cancer Cell. 2012; 21:642-654.

15. Fukuda A, Wang SC, Morris JPt, Folias AE, Liou A, Kim GE, Akira S, Boucher KM, Firpo MA, Mulvihill SJ, Hebrok M. Stat3 and MMP7 contribute to pancreatic ductal adenocarcinoma initiation and progression. Cancer Cell. 2011; 19:441-455.

16. Hedvat M, Huszar D, Herrmann A, Gozgit JM, Schroeder A, Sheehy A, Buettner R, Proia D, Kowolik CM, Xin H, Armstrong B, Bebernitz G, Weng S, et al. The JAK2 inhibitor AZD1480 potently blocks Stat3 signaling and oncogenesis in solid tumors. Cancer Cell. 2009; 16:487-497.

17. Sen M, Thomas SM, Kim S, Yeh JI, Ferris RL, Johnson JT, Duvvuri U, Lee J, Sahu N, Joyce S, Freilino ML, Shi H, Li C, et al. First-in-human trial of a STAT3 decoy oligonucleotide in head and neck tumors: implications for cancer therapy. Cancer Discov. 2012; 2:694-705.

18. Hussain SF, Kong LY, Jordan J, Conrad C, Madden T, Fokt I, Priebe W, Heimberger AB. A novel small molecule inhibitor of signal transducers and activators of transcription 3 reverses immune tolerance in malignant glioma patients. Cancer Res. 2007; 67:9630-9636.

19. Lin L, Hutzen B, Zuo M, Ball S, Deangelis S, Foust E, Pandit B, Ihnat MA, Shenoy SS, Kulp S, Li PK, Li C, Fuchs J, Lin J. Novel STAT3 phosphorylation inhibitors exhibit potent growth-suppressive activity in pancreatic and breast cancer cells. Cancer Res. 2010; 70:2445-2454.

20. Yan S, Li Z, Thiele CJ. Inhibition of STAT3 with orally active JAK inhibitor, AZD1480, decreases tumor growth in Neuroblastoma and Pediatric Sarcomas In vitro and In vivo. Oncotarget. 2013; 4:433-445. doi: 10.18632/oncotarget.930.

21. Siddiquee K, Zhang S, Guida WC, Blaskovich MA, Greedy B, Lawrence HR, Yip ML, Jove R, McLaughlin MM, Lawrence NJ, Sebti SM, Turkson J. Selective chemical probe inhibitor of Stat3, identified through structure-based virtual screening, induces antitumor activity. Proc Natl Acad Sci U S A. 2007; 104:7391-7396.

22. Chatterjee D, Sabo E, Tavares R, Resnick MB. Inverse association between Raf Kinase Inhibitory Protein and signal transducers and activators of transcription 3 expression in gastric adenocarcinoma patients: implications for clinical outcome. Clin Cancer Res. 2008; 14:2994-3001.

23. Deng J, Liang H, Zhang R, Sun D, Pan Y, Liu Y, Zhang L, Hao X. STAT3 is associated with lymph node metastasis in gastric cancer. Tumour Biol. 2013; 34:2791-2800.

24. Deng JY, Sun D, Liu XY, Pan Y, Liang H. STAT-3 correlates with lymph node metastasis and cell survival in gastric cancer. World J Gastroenterol. 2010; 16:5380-5387.

25. Jia Y, Liu D, Xiao D, Ma X, Han S, Zheng Y, Sun S, Zhang M, Gao H, Cui X, Wang Y. Expression of 
AFP, STAT3 is involved in arsenic trioxide-induced apoptosis and inhibition of proliferation in AFP-producing gastric cancer cells. PLoS One. 2013; 8:e54774.

26. Kim DY, Cha ST, Ahn DH, Kang HY, Kwon CI, Ko KH, Hwang SG, Park PW, Rim KS, Hong SP. STAT3 expression in gastric cancer indicates a poor prognosis. J Gastroenterol Hepatol. 2009; 24:646-651.

27. Lee J, Kang WK, Park JO, Park SH, Park YS, Lim HY, Kim J, Kong J, Choi MG, Sohn TS, Noh JH, Bae JM, Kim S, et al. Expression of activated signal transducer and activator of transcription 3 predicts poor clinical outcome in gastric adenocarcinoma. Apmis. 2009; 117:598-606.

28. Xiong H, Du W, Wang J-L, Wang Y-C, Tang J-T, Hong J, Fang J-Y. Constitutive activation of STAT3 is predictive of poor prognosis in human gastric cancer. J Mol Med (Berl). 2012; 90:1037-1046.

29. Yakata Y, Nakayama T, Yoshizaki A, Kusaba T, Inoue K, Sekine I. Expression of p-STAT3 in human gastric carcinoma: significant correlation in tumour invasion and prognosis. Int J Oncol. 2007; 30:437-442.

30. Ai T, Wang Z, Zhang M, Zhang L, Wang N, Li W, Song L. Expression and prognostic relevance of STAT3 and cyclin D1 in non-small cell lung cancer. Int J Biol Markers. 2012; 27:e132-138.

31. Li X, Yu Z, Li Y, Liu S, Gao C, Hou X, Yao R, Cui L. The tumor suppressor miR-124 inhibits cell proliferation by targeting STAT3 and functions as a prognostic marker for postoperative NSCLC patients. Int J Oncol. 2015; 46:798-808.

32. van Cruijsen H, Ruiz MG, van der Valk P, de Gruijl TD, Giaccone G. Tissue micro array analysis of ganglioside N-glycolyl GM3 expression and signal transducer and activator of transcription (STAT)-3 activation in relation to dendritic cell infiltration and microvessel density in nonsmall cell lung cancer. BMC Cancer. 2009; 9:180.

33. Wang $M$, Chen GY, Song HT, Hong X, Yang ZY, Sui GJ. Significance of CXCR4, phosphorylated STAT3 and VEGF-A expression in resected non-small cell lung cancer. Exp Ther Med. 2011; 2:517-522.

34. Yin Z, Zhang Y, Li Y, Lv T, Liu J, Wang X. Prognostic significance of STAT3 expression and its correlation with chemoresistance of non-small cell lung cancer cells. Acta Histochem. 2012; 114:151-158.

35. Yu Y, Zhao Q, Wang Z, Liu XY. Activated STAT3 correlates with prognosis of non-small cell lung cancer and indicates new anticancer strategies. Cancer Chemother Pharmacol. 2015; 75:917-922.

36. Zhang W, Pal SK, Liu X, Yang C, Allahabadi S, Bhanji S, Figlin RA, Yu H, Reckamp KL. Myeloid clusters are associated with a pro-metastatic environment and poor prognosis in smoking-related early stage non-small cell lung cancer. PLoS One. 2013; 8:e65121.

37. Zhao X, Sun X, Li XL. Expression and clinical significance of STAT3, P-STAT3, and VEGF-C in small cell lung cancer.
Asian Pacific journal of cancer prevention: Asian Pac J Cancer Prev. 2012; 13:2873-2877.

38. Abou-Ghazal M, Yang DS, Qiao W, Reina-Ortiz C, Wei J, Kong L-Y, Fuller GN, Hiraoka N, Priebe W, Sawaya R, Heimberger AB. The incidence, correlation with tumorinfiltrating inflammation, and prognosis of phosphorylated STAT3 expression in human gliomas. Clin Cancer Res. 2008; 14:8228-8235.

39. Birner P, Toumangelova-Uzeir K, Natchev S, Guentchev M. STAT3 tyrosine phosphorylation influences survival in glioblastoma. J Neurooncol. 2010; 100:339-343.

40. Lin GS, Yang LJ, Wang XF, Chen YP, Tang WL, Chen L, Lin ZX. STAT3 Tyr705 phosphorylation affects clinical outcome in patients with newly diagnosed supratentorial glioblastoma. Med Oncol. 2014; 31:924.

41. Slinger E, Maussang D, Schreiber A, Siderius M, Rahbar A, Fraile-Ramos A, Lira SA, SoderbergNaucler C, Smit MJ. HCMV-encoded chemokine receptor US28 mediates proliferative signaling through the IL-6STAT3 axis. Sci Signal. 2010; 3:ra58.

42. Wang Y, Chen L, Bao Z, Li S, You G, Yan W, Shi Z, Liu Y, Yang P, Zhang W, Han L, Kang C, Jiang T. Inhibition of STAT3 reverses alkylator resistance through modulation of the AKT and beta-catenin signaling pathways. Oncol Rep. $2011 ; 26: 1173-1180$.

43. Kusaba T, Nakayama T, Yamazumi K, Yakata Y, Yoshizaki A, Inoue K, Nagayasu T, Sekine I. Activation of STAT3 is a marker of poor prognosis in human colorectal cancer. Oncol Rep. 2006; 15:1445-1451.

44. Min $\mathrm{H}$, Wei-hong $\mathrm{Z}$. Constitutive activation of signal transducer and activator of transcription 3 in epithelial ovarian carcinoma. J Obstet Gynaecol Res. 2009; 35:918-925.

45. Takemoto S, Ushijima K, Kawano K, Yamaguchi T, Terada A, Fujiyoshi N, Nishio S, Tsuda N, Ijichi M, Kakuma T, Kage M, Hori D, Kamura T. Expression of activated signal transducer and activator of transcription-3 predicts poor prognosis in cervical squamous-cell carcinoma. Br J Cancer. 2009; 101:967-972.

46. Mano Y, Aishima S, Fujita N, Tanaka Y, Kubo Y, Motomura T, Taketomi A, Shirabe K, Maehara Y, Oda Y. Tumor-associated macrophage promotes tumor progression via STAT3 signaling in hepatocellular carcinoma. Pathobiology. 2013; 80:146-154.

47. Zhang CH, Xu GL, Jia WD, Li JS, Ma JL, Ren WH, Ge YS, Yu JH, Liu WB, Wang W. Activation of STAT3 signal pathway correlates with twist and E-cadherin expression in hepatocellular carcinoma and their clinical significance. J Surg Res. 2012; 174:120-129.

48. Wu Z-S, Cheng X-W, Wang X-N, Song N-J. Prognostic significance of phosphorylated signal transducer and activator of transcription 3 and suppressor of cytokine signaling 3 expression in human cutaneous melanoma. Melanoma Res. 2011; 21:483-490. 
49. Schoppmann SF, Jesch B, Friedrich J, Jomrich G, Maroske F, Birner P. Phosphorylation of signal transducer and activator of transcription 3 (STAT3) correlates with Her2 status, carbonic anhydrase 9 expression and prognosis in esophageal cancer. Clin Exp Metastasis. 2012; 29:615-624.

50. Ryu K, Choy E, Yang C, Susa M, Hornicek FJ, Mankin H, Duan Z. Activation of signal transducer and activator of transcription 3 (Stat3) pathway in osteosarcoma cells and overexpression of phosphorylated-Stat 3 correlates with poor prognosis. J Orthop Res. 2010; 28:971-978.

51. Wang YC, Zheng LH, Ma BA, Zhou Y, Zhang MH, Zhang DZ, Fan QY. Clinical value of signal transducers and activators of transcription 3 (STAT3) gene expression in human osteosarcoma. Acta Histochem. 2011; 113:402-408.

52. Denley SM, Jamieson NB, McCall P, Oien KA, Morton JP, Carter CR, Edwards J, McKay CJ. Activation of the IL$6 \mathrm{R} / \mathrm{Jak} / \mathrm{stat}$ pathway is associated with a poor outcome in resected pancreatic ductal adenocarcinoma. J Gastrointest Surg. 2013; 17:887-898.

53. Huang C, Huang R, Chang W, Jiang T, Huang K, Cao J, Sun X, Qiu Z. The expression and clinical significance of pSTAT3, VEGF and VEGF-C in pancreatic adenocarcinoma. Neoplasma. 2012; 59:52-61.

54. Li C, Wang Z, Liu Y, Wang P, Zhang R. STAT3 expression correlates with prognosis of thymic epithelial tumors. J Cardiothorac Surg. 2013; 8:92.

55. Piperi C, Samaras V, Levidou G, Kavantzas N, Boviatsis E, Petraki K, Grivas A, Barbatis C, Varsos V, Patsouris E, Korkolopoulou P. Prognostic significance of IL-8-STAT-3 pathway in astrocytomas: correlation with IL-6, VEGF and microvessel morphometry. Cytokine. 2011; 55:387-395.

56. Zhao Y, Zhang J, Xia H, Zhang B, Jiang T, Wang J, Chen X, Wang Y. Stat 3 is involved in the motility, metastasis and prognosis in lingual squamous cell carcinoma. Cell Biochem Funct. 2012; 30:340-346.

57. Chen $\mathrm{CC}$, Chen WC, Lu CH, Wang WH, Lin PY, Lee KD, Chen MF. Significance of interleukin-6 signaling in the resistance of pharyngeal cancer to irradiation and the epidermal growth factor receptor inhibitor. Int J Radiat Oncol Biol Phys. 2010; 76:1214-1224.

58. Tam L, McGlynn LM, Traynor P, Mukherjee R, Bartlett JM, Edwards J. Expression levels of the JAK/STAT pathway in the transition from hormone-sensitive to hormone-refractory prostate cancer. Br J Cancer. 2007; 97:378-383.

59. Horiguchi A, Oya M, Shimada T, Uchida A, Marumo K, Murai M. Activation of signal transducer and activator of transcription 3 in renal cell carcinoma: a study of incidence and its association with pathological features and clinical outcome. J Urol. 2002; 168:762-765.

60. Zhang LJ, Liu W, Gao YM, Qin YJ, Wu RD. The expression of IL-6 and STAT3 might predict progression and unfavorable prognosis in Wilms' tumor. Biochem Biophys Res Commun. 2013; 435:408-413.
61. Sonnenblick A, Uziely B, Nechushtan H, Kadouri L, Galun E, Axelrod JH, Katz D, Daum H, Hamburger T, Maly B, Allweis TM, Peretz T. Tumor STAT3 tyrosine phosphorylation status, as a predictor of benefit from adjuvant chemotherapy for breast cancer. Breast Cancer Res Treat. 2013; 138:407-413.

62. Sheen-Chen S-M, Huang C-C, Tang R-P, Chou F-F, Eng H-L. Prognostic value of signal transducers and activators of transcription 3 in breast cancer. Cancer Epidemiol Biomarkers Prev. 2008; 17:2286-2290.

63. Dolled-Filhart M, Camp RL, Kowalski DP, Smith BL, Rimm DL. Tissue microarray analysis of signal transducers and activators of transcription 3 (Stat3) and phospho-Stat3 (Tyr705) in node-negative breast cancer shows nuclear localization is associated with a better prognosis. Clin Cancer Res. 2003; 9:594-600.

64. Sonnenblick A, Shriki A, Galun E, Axelrod JH, Daum H, Rottenberg Y, Hamburger T, Mali B, Peretz T. Tissue microarray-based study of patients with lymph nodepositive breast cancer shows tyrosine phosphorylation of signal transducer and activator of transcription 3 (tyrosine705-STAT3) is a marker of good prognosis. Clin Transl Oncol. 2012; 14:232-236.

65. Yamashita H, Nishio M, Ando Y, Zhang Z, Hamaguchi M, Mita K, Kobayashi S, Fujii Y, Iwase H. Stat5 expression predicts response to endocrine therapy and improves survival in estrogen receptor-positive breast cancer. Endocr Relat Cancer. 2006; 13:885-893.

66. Woo S, Lee BL, Yoon J, Cho SJ, Baik TK, Chang MS, Lee HE, Park JW, Kim YH, Kim WH. Constitutive activation of signal transducers and activators of transcription 3 correlates with better prognosis, cell proliferation and hypoxia-inducible factor-1alpha in human gastric cancer. Pathobiology. 2011; 78:295-301.

67. Cortas T, Eisenberg R, Fu P, Kern J, Patrick L, Dowlati A. Activation state EGFR and STAT-3 as prognostic markers in resected non-small cell lung cancer. Lung Cancer. 2007; 55:349-355.

68. Galleges Ruiz MI, Floor K, Steinberg SM, Grunberg K, Thunnissen FBJM, Belien JAM, Meijer GA, Peters GJ, Smit EF, Rodriguez JA, Giaccone G. Combined assessment of EGFR pathway-related molecular markers and prognosis of NSCLC patients. Br J Cancer. 2009; 100:145-152.

69. Haura EB, Zheng Z, Song L, Cantor A, Bepler G. Activated epidermal growth factor receptor-Stat-3 signaling promotes tumor survival in vivo in non-small cell lung cancer. Clin Cancer Res. 2005; 11:8288-8294.

70. Gordziel C, Bratsch J, Moriggl R, Knosel T, Friedrich $\mathrm{K}$. Both STAT1 and STAT3 are favourable prognostic determinants in colorectal carcinoma. Br J Cancer. 2013; 109:138-146.

71. Monnien F, Zaki H, Borg C, Mougin C, Bosset JF, Mercier M, Arbez-Gindre F, Kantelip B. Prognostic value of phosphorylated STAT3 in advanced rectal cancer: a 
study from 104 French patients included in the EORTC 22921 trial. J Clin Pathol. 2010; 63:873-878.

72. Lee I, Fox PS, Ferguson SD, Bassett R, Kong LY, Schacherer CW, Gershenwald JE, Grimm EA, Fuller GN, Heimberger AB. The expression of p-STAT3 in stage IV melanoma: risk of CNS metastasis and survival. Oncotarget. 2012; 3:336-344. doi: 10.18632/oncotarget.475.

73. Hbibi AT, Lagorce C, Wind P, Spano JP, Des Guetz G, Milano G, Benamouzig R, Rixe O, Morere JF, Breau JL, Martin A, Fagard R. Identification of a functional EGF-R/ p60c-src/STAT3 pathway in colorectal carcinoma: analysis of its long-term prognostic value. Cancer Biomark. 2008; 4:83-91.

74. Rosen DG, Mercado-Uribe I, Yang G, Bast RC, Jr., Amin HM, Lai R, Liu J. The role of constitutively active signal transducer and activator of transcription 3 in ovarian tumorigenesis and prognosis. Cancer. 2006; 107:2730-2740.

75. Yang C, Lee H, Jove V, Deng J, Zhang W, Liu X, Forman S, Dellinger TH, Wakabayashi M, Yu H, Pal S. Prognostic significance of B-cells and pSTAT3 in patients with ovarian cancer. PLoS One. 2013; 8:e54029.

76. Chen HH, Chou CY, Wu YH, Hsueh WT, Hsu CH, Guo HR, Lee WY, Su WC. Constitutive STAT5 activation correlates with better survival in cervical cancer patients treated with radiation therapy. Int J Radiat Oncol Biol Phys. 2012; 82:658-666.

77. Choi CH, Song SY, Kang H, Lee Y-Y, Kim C-J, Lee J-W, Kim T-J, Kim B-G, Lee J-H, Bae D-S. Prognostic significance of p-STAT3 in patients with bulky cervical carcinoma undergoing neoadjuvant chemotherapy. The J Obstet Gynaecol Res. 2010; 36:304-310.

78. You Z, Xu D, Ji J, Guo W, Zhu W, He J. JAK/STAT signal pathway activation promotes progression and survival of human oesophageal squamous cell carcinoma. Clin Transl Oncol. 2012; 14:143-149.

79. Chang $\mathrm{KC}, \mathrm{Wu} \mathrm{MH}$, Jones $\mathrm{D}$, Chen FF, Tseng YL. Activation of STAT3 in thymic epithelial tumours correlates with tumour type and clinical behaviour. J Pathol. 2006; 210:224-233.

80. Shah NG, Trivedi TI, Tankshali RA, Goswami JA, Jetly DH, Kobawala TP, Shukla SN, Shah PM, Verma RJ. Stat3 expression in oral squamous cell carcinoma: association with clinicopathological parameters and survival. Int J Biol Markers. 2006; 21:175-183.

81. Macha MA, Matta A, Kaur J, Chauhan SS, Thakar A, Shukla NK, Gupta SD, Ralhan R. Prognostic significance of nuclear pSTAT3 in oral cancer. Head Neck. 2011; 33:482-489.

82. Yang C, Schwab JH, Schoenfeld AJ, Hornicek FJ, Wood KB, Nielsen GP, Choy E, Mankin H, Duan Z. A novel target for treatment of chordoma: signal transducers and activators of transcription 3. Mol Cancer Ther. 2009; 8:2597-2605.
83. Pectasides E, Egloff A-M, Sasaki C, Kountourakis P, Burtness B, Fountzilas G, Dafni U, Zaramboukas T, Rampias T, Rimm D, Grandis J, Psyrri A. Nuclear localization of signal transducer and activator of transcription 3 in head and neck squamous cell carcinoma is associated with a better prognosis. Clin Cancer Res. 2010; 16:2427-2434.

84. Kim Y-J, Go H, Wu H-G, Jeon YK, Park SW, Lee SH. Immunohistochemical study identifying prognostic biomolecular markers in nasopharyngeal carcinoma treated by radiotherapy. Head Neck. 2011; 33:1458-1466.

85. Bharadwaj U, Eckols TK, Kolosov M, Kasembeli MM, Adam A, Torres D, Zhang X, Dobrolecki LE, Wei W, Lewis MT, Dave B, Chang JC, Landis MD, et al. Drugrepositioning screening identified piperlongumine as a direct STAT3 inhibitor with potent activity against breast cancer. Oncogene. 2015; 34:1341-1353.

86. Zhang X, Yue P, Page BD, Li T, Zhao W, Namanja AT, Paladino D, Zhao J, Chen Y, Gunning PT, Turkson J. Orally bioavailable small-molecule inhibitor of transcription factor Stat3 regresses human breast and lung cancer xenografts. Proc Natl Acad Sci U S A. 2012; 109:9623-9628.

87. Kong LY, Abou-Ghazal MK, Wei J, Chakraborty A, Sun W, Qiao W, Fuller GN, Fokt I, Grimm EA, Schmittling RJ, Archer GE, Jr., Sampson JH, Priebe W, et al. A novel inhibitor of signal transducers and activators of transcription 3 activation is efficacious against established central nervous system melanoma and inhibits regulatory $\mathrm{T}$ cells. Clin Cancer Res. 2008; 14:5759-5768.

88. Rath KS, Naidu SK, Lata P, Bid HK, Rivera BK, McCann GA, Tierney BJ, Elnaggar AC, Bravo V, Leone G, Houghton P, Hideg K, Kuppusamy P, et al. HO-3867, a safe STAT3 inhibitor, is selectively cytotoxic to ovarian cancer. Cancer Res. 2014; 74:2316-2327.

89. Liberati A, Altman DG, Tetzlaff J, Mulrow C, Gotzsche PC, Ioannidis JP, Clarke M, Devereaux PJ, Kleijnen J, Moher D. The PRISMA statement for reporting systematic reviews and meta-analyses of studies that evaluate health care interventions: explanation and elaboration. PLoS Med. 2009; 6:e1000100.

90. Stang A. Critical evaluation of the Newcastle-Ottawa scale for the assessment of the quality of nonrandomized studies in meta-analyses. Eur J Epidemiol. 2010; 25:603-605.

91. Deeks JJ HJ, Altman DG. Analysing and presenting results. In: Higgins JPT, Green S, ed. Cochrane Handbook for Systematic Reviews of Interventions 425 Chichester, UK: John Wiley \& Sons. 2006. 